\title{
Hyperbolic geometry and non-Kähler manifolds with trivial canonical bundle
}

\author{
JOEL Fine \\ DMITRI PANOV
}

\begin{abstract}
We use hyperbolic geometry to construct simply connected symplectic or complex manifolds with trivial canonical bundle and with no compatible Kähler structure. We start with the desingularisations of the quadric cone in $\mathbb{C}^{4}$ : the smoothing is a natural $S^{3}$-bundle over $H^{3}$, its holomorphic geometry is determined by the hyperbolic metric; the small-resolution is a natural $S^{2}$-bundle over $H^{4}$ with symplectic geometry determined by the metric. Using hyperbolic geometry, we find orbifold quotients with trivial canonical bundle; smooth examples are produced via crepant resolutions. In particular, we find the first example of a simply connected symplectic 6-manifold with $c_{1}=0$ that does not admit a compatible Kähler structure. We also find infinitely many distinct complex structures on $2\left(S^{3} \times S^{3}\right) \#\left(S^{2} \times S^{4}\right)$ with trivial canonical bundle. Finally, we explain how an analogous construction for hyperbolic manifolds in higher dimensions gives symplectic non-Kähler "Fano" manifolds of dimension 12 and higher.
\end{abstract}

53D35, 32Q55; 51M10, 57M25

\section{Introduction}

The main goal of this article is to describe how hyperbolic geometry can be used to construct simply connected complex and symplectic manifolds with trivial canonical bundle, which admit no compatible Kähler structure. In the symplectic case, trivial means $c_{1}=0$, whilst in the complex case, trivial means holomorphically trivial. Our examples have real dimension six. Hyperbolic geometry in dimension three gives complex examples whilst hyperbolic geometry in dimension four leads to symplectic examples.

Using this approach we find, amongst other things the following kind of examples.

Theorem 1 There exists a compact simply connected 6-manifold $X$ which admits a symplectic structure with $c_{1}=0$. Moreover, there is no Kähler metric on $X$ which has $c_{1}=0$.

To the best of our knowledge, this is the first such 6-dimensional example. 
Theorem 2 There are infinitely many distinct complex structures on $2\left(S^{3} \times S^{3}\right)$ \# $\left(S^{2} \times S^{4}\right)$ all with trivial canonical bundle.

These examples are necessarily non-Kähler because all degree 2 cohomology classes on $2\left(S^{3} \times S^{3}\right) \#\left(S^{2} \times S^{4}\right)$ have zero top power.

Before giving an outline of the constructions, we begin by recalling some background.

\subsection{Non-Kähler examples in dimension four}

First consider the case of real dimension four. It follows from the classification of compact complex surfaces that the only simply connected compact complex surfaces with trivial canonical bundle are K3 surfaces and so, in particular, are Kähler. In the symplectic case the situation is not yet understood, but it is a folklore conjecture that Kähler K3 surfaces again provide the only examples. So, conjecturally, in dimension four all simply connected symplectic manifolds with $c_{1}=0$ are Kähler too. If we believe this conjecture, to find the first non-Kähler examples of simply connected manifolds with trivial canonical bundle, we should look to higher dimensions.

\subsection{Non-Kähler examples in dimension six}

In dimension six, much use has been made of ordinary double-points and their desingularisations. An ordinary double-point is modelled locally on a neighbourhood of the origin in the quadric cone $\sum z_{j}^{2}=0$ in $\mathbb{C}^{4}$. To desingularise the cone, one can smooth it to obtain the smooth affine quadric $\sum z_{j}^{2}=1$; this replaces the double point by a three-sphere. Alternatively, one can resolve the double-point by the so-called small resolution, which is the total space of $\mathcal{O}(-1) \oplus \mathcal{O}(-1) \rightarrow \mathbb{C P}^{1}$; here the double point is replaced by a two-sphere. (We will give more detail about these desingularisations later in Section 2.) Note that the double point and both desingularisations have trivial canonical bundle.

In the complex setting this approach was used by Friedman [16] and Tian [41], following ideas of Clemens [8], to produce complex manifolds with trivial canonical bundle. Starting from a complex manifold $X$ with trivial canonical bundle and ordinary double points they considered the manifold $X^{\prime}$ obtained by smoothing all the double points. Under certain conditions, the complex structure on $X$ can be smoothed to give a complex structure on $X^{\prime}$, again with trivial canonical bundle. In this way one finds a family of complex structures on $n\left(S^{3} \times S^{3}\right)$ for all $n \geq 2$, with trivial canonical bundle. (The article of Lu-Tian [28] explains the details for these particular smoothings.) These manifolds are clearly not Kähler. 
There is an analogous story on the symplectic side, explained in the article of SmithThomas-Yau [39]. Here, one starts with a symplectic manifold $Y$ with ordinary double points and trivial canonical bundle and considers the manifold $Y^{\prime}$ obtained by the small resolution of all the double points. Under certain conditions, the symplectic structure on $Y$ can be resolved to give a symplectic structure on $Y^{\prime}$ with $c_{1}=0$. In this way SmithThomas-Yau produced many interesting examples of simply connected symplectic manifolds with $c_{1}=0$ which are widely believed to be non-Kähler. However, in contrast with the complex side, they were unable to find examples which violate the standard Kähler topological restrictions. It is still unknown if any of the examples they give actually are non-Kähler.

\subsection{Non-Kähler examples in dimension $4 n$}

In [21], Guan constructs examples of simply connected compact complex-symplectic manifolds of dimension $4 n$ for $n \geq 2$, which do not admit a compatible Kähler structure. (See also the article [5] of Bogomolov for an alternative exposition of Guan's construction.) Taking the real part of the complex symplectic form, one obtains a symplectic manifold in the real sense which has $c_{1}=0$. In [21], Guan proves that his examples are not diffeomorphic to hyperkähler manifolds. In fact even more is true; whilst it is not proven directly in [21], Guan has informed us that it follows from the results of [21] that his manifolds admit no Kähler structure whatsoever.

Guan's construction uses Kodaira-Thurston surfaces to produce a single example of a simply connected $4 n$-manifold for each $n \geq 2$ which admits complex-symplectic structures. One might think of Guan's construction as a non-Kähler analogue of the infinite series of hyperkähler manifolds coming from the Hilbert scheme of $n$ points on a hyperkähler surface. In contrast, the construction we present here produces large numbers of topologically distinct symplectic manifolds all of the same dimension. Whilst we describe just one in this article, we produce examples with arbitrarily high Betti numbers in a sequel [14]. The idea is outlined here in Section 6.

\subsection{A hyperbolic picture of the desingularisations}

As in the articles $[8 ; 16 ; 41 ; 39]$ mentioned above, the construction of symplectic and complex manifolds that we explore in this article relies on the desingularisations of the threefold quadric cone. However, rather than using the small resolution or smoothing as local models for desingularising double-points, here we consider their global geometry.

1.4.1 $H^{4}$ and the small resolution As we explain in Section 2.3, the symplectic geometry of the small resolution $R=\mathcal{O}(-1) \oplus \mathcal{O}(-1)$ is intimately related to hyperbolic geometry in dimension four. $R$ is a natural $S^{2}$-bundle over $H^{4}$, with the 
symplectic Calabi-Yau structure on $R$ determined by the metric on $H^{4}$. Indeed, $R$ is symplectomorphic to the twistor space of $H^{4}$; the symplectic structure on the twistor space was first defined by Reznikov [36] and Davidov-Muškarov-Grantcharov [10] and a symplectomorphism with $R$ was given by the authors in [15]. In Section 2.3.2 and Section 2.3.3 we give two additional descriptions: symplectically, $R$ can be seen as a coadjoint orbit of $\mathrm{SO}(4,1)$, or as a certain pseudo-Kähler manifold, via a construction involving quaternions, analogous to the twistor fibration $\mathbb{C P}^{3} \rightarrow \mathbb{H} \mathbb{P}^{1} \cong S^{4}$. It follows that any hyperbolic 4-manifold carries an $S^{2}$-bundle-its twistor space-whose total space is a symplectic manifold with trivial canonical bundle.

To produce a simply connected example, in Section 4 we use a version of the Kummer construction. Recall that in the Kummer construction, one considers the quotient of an abelian surface by the central involution $z \mapsto-z$. Resolving the 16 orbifold points gives a K3 surface.

The role of the abelian surface in our situation is played by a beautiful hyperbolic 4-manifold called the Davis manifold [11] (see also the description in RatcliffeTschantz [34]). This manifold is constructed by gluing opposite 3-faces of a 120-cell in $H^{4}$, a certain regular four-dimensional polyhedron (so-called because it has 120 three-dimensional faces, each a regular dodecahedron).

An important point for our purposes is that reflection in the centre of the 120-cell gives an isometry of the Davis manifold $M$. This isometry gives a quotient $M / \mathbb{Z}_{2}$ which is a simply connected singular manifold with 122 isolated singularities modelled on $z \mapsto-z$, just as in the Kummer construction. In terms of the symplectic $S^{2}$-bundle $Z \rightarrow M$, the isometry induces a symplectic action of $\mathbb{Z}_{2}$ on $Z$ which fixes 122 fibres. The quotient is a symplectic orbifold with trivial canonical bundle, with singularities along 122 2-spheres. Making a symplectic crepant resolution of these singularities gives a smooth simply connected symplectic manifold $\hat{Z}$. As we will see, $b_{3}(\hat{Z})=0$, which can never happen for a simply connected Kähler manifold with $c_{1}=0$.

1.4.2 $H^{3}$ and the smoothing There is an analogous story for the smoothing $S=$ $\left\{\sum z_{j}^{2}=1\right\}$, this time relating the complex geometry of $S$ with hyperbolic geometry in dimension three. $S$ is a natural $S^{3}$-bundle over $H^{3}$ with the complex geometry of $S$ determined by the metric on $H^{3}$. One way to see this is to note that $S \cong \operatorname{SL}(2, \mathbb{C})$; the action of $\mathrm{SU}(2)$ on $\operatorname{SL}(2, \mathbb{C})$ makes it a principal bundle over $\operatorname{SU}(2) \backslash \mathrm{SL}(2, \mathbb{C}) \cong H^{3}$, the principal spin bundle of $H^{3}$. As a consequence, any hyperbolic 3-manifold carries an $S^{3}$-bundle whose total space is a complex manifold with trivial canonical bundle.

To produce simply connected examples, in Section 3 we consider hyperbolic orbifold metrics on $S^{3}$ with cone angle $2 \pi / m$ along a knot $K$. It is a standard fact that there 
is a smooth hyperbolic manifold $M$ admitting an isometric $\mathbb{Z}_{m}$-action with quotient $M \rightarrow S^{3}$ branched over $K$. Now, as $M$ is hyperbolic, its spin bundles are complex manifolds with trivial canonical bundle. As we will show, provided the spin-structure $P \rightarrow M$ is well-chosen, the generator of the $\mathbb{Z}_{m}$-action lifts to $P$ where it generates a $\mathbb{Z}_{2 m}$-action (covering the $\mathbb{Z}_{m}$-action on $M$ via the projection $\mathbb{Z}_{2 m} \rightarrow \mathbb{Z}_{m}$ ).

The quotient $P / \mathbb{Z}_{2 m}$ is just the quotient of the frame bundle $Q \rightarrow M$ by the lift of the action of $\mathbb{Z}_{m}$. It is straightforward to see that $\mathbb{Z}_{m}$ acts freely on $Q$ and so $Q / \mathbb{Z}_{m}$ is smooth and has fundamental group equal to the original orbifold fundamental group of the metric on $S^{3}$. To remedy this, we "twist" the $\mathbb{Z}_{2 m}$-action on $P$ around the knot $K$; this induces fixed points in the fibres over $K$ and results in a singular but simply connected quotient of $P$. Finally, we obtain a smooth manifold by taking a crepant resolution.

The examples we find this way have a smooth surjection $X \rightarrow S^{3}$. Away from the knot, this makes $X$ a trivial fibration $S^{3} \times\left(S^{3} \backslash K\right)$. These threefolds can never be Kähler; as we explain, they admit a $\mathbb{C}^{*}$-action with no fixed points. This construction provides, amongst other things, infinitely many distinct complex structures with trivial canonical bundle on $2\left(S^{3} \times S^{3}\right) \#\left(S^{2} \times S^{4}\right)$.

\subsection{Symplectic non-Kähler "Fano" manifolds}

In Section 5 we outline a relationship between symplectic and hyperbolic geometry in all even dimensions: a hyperbolic manifold of dimension $2 n$ is the base of a fibre bundle whose total space is a symplectic manifold of real dimension $n(n+1)$. (The fibre bundle is the twistor space; these symplectic manifolds were first considered by Reznikov [36].) When $n=1$ the fibres are zero-dimensional and the total space is just the original surface. In this case, the symplectic manifold is "general type" with symplectic class a negative multiple of $c_{1}$. When $n=2$, we have the situation described above, for which $c_{1}=0$. For all higher dimensions, however, it turns out that the symplectic manifold has symplectic class equal to a positive multiple of $c_{1}$. No compact example produced this way can be Kähler (eg, for fundamental group reasons). To the best of our knowledge, these examples (found first by Reznikov) are the first symplectic non-Kähler manifolds which have symplectic class equal to a positive multiple of $c_{1}$, symplectic analogues of Fano varieties.

It is interesting to compare this with the situation in dimension four. There, it is a consequence of the work of Gromov [20], Taubes [40] and McDuff [30] that a symplectic Fano 4-manifold must be Kähler. Meanwhile, the lowest dimension attained by our construction is twelve. It is natural to ask for the minimal dimension in which nonKähler examples occur. Do they exist in dimension six? The symplectic Fanos coming 
from hyperbolic $2 n$-manifolds also have the property that $c_{1}^{n}$ can be arbitrarily large (it is essentially the volume of the hyperbolic $2 n$-manifold). Again, it seems natural to ask for the minimal dimension in which Fano manifolds exist for which $c_{1}^{n}$ can be made arbitrarily large.

Acknowledgements It is a pleasure to thank the following people for discussions held during the course of this work: Mohammed Abouzaid, Michel Boileau, Alessio Corti, Simon Donaldson, Allan Edmonds, Daniel Guan, Maxim Kontsevich, Federica Pasquotto, Vicente Muñoz, Brendan Owens, Simon Salamon, Paul Seidel, Ivan Smith, Burt Totaro, Richard Thomas, Henry Wilton, Claire Voisin, Jean-Yves Welschinger and Dominic Wright. We would also like to thank an anonymous reader who pointed out an error in an earlier version of this work.

The first author was supported by an FNRS chargé de recherche fellowship. The second author was supported by EPSRC grant EP/E044859/1.

\section{Hyperbolic geometry and the conifold}

\subsection{Desingularisations of the conifold}

Fix a nondegenerate complex quadratic form $q$ on $\mathbb{C}^{4}$. The conifold is the affine quadric cone $Q=\{q(\zeta)=0\}$. We will consider two well-studied ways to remove the singularity at the origin, giving smooth Kähler manifolds with trivial canonical bundle. We describe this briefly here; for more details we refer to [39], from where we originally learnt this material.

One desingularisation is the smooth affine quadric $S=\{q(\zeta)=1\}$, called the smoothing of $Q . S$ is a Kähler manifold with trivial canonical bundle, although we will be concerned only with the complex geometry of $S$.

Another way to desingularise the conifold is to take a resolution. Choose coordinates $(x, y, w, z)$ so that the quadratic form is given by $q(x, y, z, w)=,x w-y z$. Let $R$ denote the total space of $\pi: \mathcal{O}(-1) \oplus \mathcal{O}(-1) \rightarrow \mathbb{C P}^{1}$. Each summand of $R$ has a natural map $\mathcal{O}(-1) \rightarrow \mathbb{C}^{2}$; these combine to give a map $p: R \rightarrow \mathbb{C}^{2} \oplus \mathbb{C}^{2} \cong \mathbb{C}^{4}$. Here, we use the identification $((a, b),(c, d)) \mapsto(a, b, c, d)$. The image of $p$ consists of points $(x, y, z, w)$ such that $[x: y]=[z: w]$, ie, such that $x w-y z=0$, which is the conifold. So $p: R \rightarrow Q$ is a resolution, called the small resolution, in which the singularity has been replaced by the zero section in $R$.

In this description, we could equally have chosen to identify $\mathbb{C}^{2} \oplus \mathbb{C}^{2} \cong \mathbb{C}^{4}$ by $((a, b),(c, d)) \mapsto(a, c, b, d)$. With this choice, the image of $p: R \rightarrow \mathbb{C}^{4}$ consists of 
points $(x, y, z, w)$ such that $[x: z]=[y: w]$ which is again the conifold $x w-y z=0$. So, in fact, there are two inequivalent ways to view $R$ as the small resolution of $Q$. Put another way, there are two small resolutions $R_{ \pm} \rightarrow Q$; each is isomorphic to $\mathcal{O}(-1) \oplus \mathcal{O}(-1)$ abstractly, but there is no isomorphism between them which respects the projections to $Q$.

There is an alternative coordinate-free description of the small resolutions. Blow up the origin in $\mathbb{C}^{4}$ to obtain $\overline{\mathbb{C}}^{4}$. The proper transform $\widetilde{Q}$ of $Q$ meets the exceptional $\mathbb{C P}^{3} \subset \overline{\mathbb{C}}^{4}$ in a quadric surface. This surface is biholomorphic to $\mathbb{C P}^{1} \times \mathbb{C P}^{1}$ and each of the rulings has negative normal bundle. Blowing down one or other of the rulings gives the two small resolutions $R_{ \pm} \rightarrow Q$ of the conifold.

Either resolution $R$ is a Kähler manifold with trivial canonical bundle, although we will be concerned only with the symplectic geometry of $R$. The symplectic form is given by

$$
\omega_{R}=\pi^{*} \omega_{\mathbb{C P}^{1}}+p^{*} \omega_{\mathbb{C}^{4}}
$$

where $\pi: R \rightarrow \mathbb{C P}^{1}$ and $p: R \rightarrow Q \subset \mathbb{C}^{4}$ are the vector bundle and resolution projections respectively.

The first hint of a link with twistor geometry is provided by considering the symplectic action of $\mathrm{SO}(4)$ on $R$. The Hermitian metric and complex quadratic form on $\mathbb{C}^{4}$ define a choice of conjugation map (the real points are those on which the Hermitian and complex forms agree). Then $\mathrm{SO}(4)$ is the subgroup of $\mathrm{U}(4)$ which commutes with this conjugation. In this way $\mathrm{SO}(4)$ acts by Kähler isometries on the conifold $Q$ and this action lifts to a Kähler action on the small resolutions $R_{ \pm}$. The action on the exceptional $\mathbb{C P}^{1}$ in $R_{ \pm}$is given by one or other of the projections $\mathrm{SO}(4) \rightarrow \mathrm{PSU}(2)$ arising from the exceptional isomorphism

$$
\mathrm{SO}(4) \cong \frac{\mathrm{SU}(2) \times \mathrm{SU}(2)}{ \pm 1}
$$

\subsection{Hyperbolic geometry and the smoothing}

To describe the connection between hyperbolic geometry and $S$ the smoothing, use coordinates $(x, y, z, w)$ in which the quadratic form $q$ is given by $q(x, y, z, w)=$ $x w-y z$. Identify $\mathbb{C}^{4}$ with the set of complex $2 \times 2$ matrices by

$$
\left(\begin{array}{cc}
x & y \\
z & w
\end{array}\right) \mapsto(x, y, z, w) .
$$

When evaluated on a matrix $A$, the quadratic form is $q(A)=\operatorname{det} A$. Hence, in this picture, $S \cong \operatorname{SL}(2, \mathbb{C})$ is given by matrices with determinant 1 . Consider the action 
of $\operatorname{SU}(2)$ on $\operatorname{SL}(2, \mathbb{C})$ given by multiplication on the left; this makes $\operatorname{SL}(2, \mathbb{C})$ into a principal $\mathrm{SU}(2)$-bundle over the symmetric space $\mathrm{SU}(2) \backslash \mathrm{SL}(2, \mathbb{C})$ which is precisely hyperbolic space $H^{3}$. (See, for example, page 453 of Helgason [22].) In fact, the bundle $\operatorname{SL}(2, \mathbb{C}) \rightarrow H^{3}$ can be identified with the principal spin bundle of $H^{3}$. To verify this note that $\operatorname{Isom}\left(H^{3}\right) \cong \operatorname{PSL}(2, \mathbb{C})$ acts freely and transitively on the frame bundle of $H^{3}$; so PSL $(2, \mathbb{C})$ can be identified with the frame bundle of $H^{3}$ whilst its double cover $\operatorname{SL}(2, \mathbb{C})$ is identified with the principal spin bundle.

As it is a complex Lie group, $\operatorname{SL}(2, \mathbb{C})$ has a holomorphic volume form which is invariant under right-multiplication. It will be essential to us that this same form is also invariant under left-multiplication; in other words, that $\operatorname{SL}(2, \mathbb{C})$ is a complex unimodular group. Given $P, Q \in \mathrm{SL}(2, \mathbb{C})$, consider the biholomorphism of $S \cong$ $\mathrm{SL}(2, \mathbb{C})$ defined by $A \mapsto P^{-1} A Q$. This sets up an isomorphism

$$
\mathrm{SO}(4, \mathbb{C}) \cong \frac{\mathrm{SL}(2, \mathbb{C}) \times \mathrm{SL}(2, \mathbb{C})}{ \pm 1}
$$

(This is, of course, just the complexification of (1).) Unimodularity of $\operatorname{SL}(2, \mathbb{C})$ now amounts to the following:

Proposition $3 \mathrm{SO}(4, \mathbb{C})$ acts by biholomorphisms on $S$. Moreover, $S$ admits an invariant holomorphic volume form.

Alternatively, the $\mathrm{SO}(4, \mathbb{C})$-invariant volume form can be seen directly, without reference to $\operatorname{SL}(2, \mathbb{C})$ and unimodularity. $\operatorname{SO}(4, \mathbb{C})$ preserves both the Euclidean holomorphic volume form $\Omega_{0}$ and the radial vector $e=x \partial_{x}+y \partial_{y}+z \partial_{z}+w \partial_{w}$, which is transverse to $S$. Hence the volume form $\Omega=\iota_{e} \Omega_{0}$ on $S$ is also invariant.

To produce compact quotients of $S$, let $M$ be an oriented compact hyperbolic 3manifold with fundamental group $\Gamma \subset \operatorname{PSL}(2, \mathbb{C})$. Since $M$ is spin (as all oriented 3 -manifolds are), $\Gamma$ lifts to $\operatorname{SL}(2, \mathbb{C})$ and the total space of the principal spin bundle of $M$ is $X=\operatorname{SL}(2, \mathbb{C}) / \Gamma$, where $\Gamma$ acts by right-multiplication. More precisely, each choice of lift of $\Gamma$ to $\operatorname{SL}(2, \mathbb{C})$ gives a spin structure on $M$; this correspondence is one-to-one: lifts and spin structures are both parametrised by $H^{1}\left(M, \mathbb{Z}_{2}\right)$. Whatever the choice of lift, the action of $\Gamma$ preserves the holomorphic volume form on $\operatorname{SL}(2, \mathbb{C})$ and so $X$ is a compact complex threefold with trivial canonical bundle.

Left-multiplication gives us additional freedom. Denote the chosen lift by $\alpha: \Gamma \rightarrow$ $\operatorname{SL}(2, \mathbb{C})$ and let $\rho: \Gamma \rightarrow \operatorname{SL}(2, \mathbb{C})$ be some other homomorphism. From $\rho$ we obtain a new action of $\Gamma$ on $S$ :

$$
\gamma \cdot A=\rho(\gamma)^{-1} A \alpha(\gamma)
$$


We will use this "twisting" to produce orbifold quotients of $S$, but it was originally used in a different context by Ghys [17]. Ghys studies infinitesimal deformations of the complex manifold $X=\operatorname{SL}(2, \mathbb{C}) / \Gamma$, where $\Gamma$ acts by right-multiplication, ie, where $\rho$ is the trivial homomorphism in the above picture. He shows that infinitesimal holomorphic deformations of $X$ are equivalent to infinitesimal deformations of the trivial homomorphism. It is possible to extend this picture, giving a description of part of the space of complex structures on $X$, something we address in forthcoming work [13].

\subsection{Hyperbolic geometry and the small resolution}

This section proves the analogue of Proposition 3 for the small resolution $R$.

Proposition $4 \mathrm{SO}(4,1)$ acts symplectomorphically on $R$, extending the action of $\mathrm{SO}(4)$. Moreover, $R$ admits an invariant compatible almost complex structure and invariant complex volume form.

Before proving this, we give three different descriptions of the hyperbolic geometry of $R$.

2.3.1 Twistorial description The twistor space of $H^{4}$ carries a natural symplectic structure (a fact noticed independently by Reznikov [36] and Davidov-MuškarovGrantcharov [10]) and this was shown to be symplectomorphic to the small resolution in [15]. We very briefly recall the idea here.

Choose coordinates $z_{j}$ on $\mathbb{C}^{4}$ in which the conifold is $\left\{\sum z_{j}^{2}=0\right\}$. The map $Q \rightarrow \mathbb{R}^{4}$ given by $z \mapsto \operatorname{Re} z$ exhibits $Q \backslash 0$ as an $S^{2}$-bundle over $\mathbb{R}^{4} \backslash 0$. The fibre over a point $x$ is all points of the form $x+i y$ where $y \in\langle x\rangle^{\perp}$ with $|y|=|x|$.

The twistor space of $\mathbb{R}^{4}$ is the bundle of unit-length self-dual two forms. Given $x \in \mathbb{R}^{4} \backslash 0$, interior contraction with $x$ gives an isomorphism $\Lambda^{+} \cong\langle x\rangle^{\perp}$. In this way we can identify $Q \backslash 0$ with the twistor space of $\mathbb{R}^{4} \backslash 0$. This identification extends over the small resolution to give an $\mathrm{SO}(4)$-equivariant identification of $R$ with the twistor space of $\mathbb{R}^{4}$. (More precisely it extends over one of the two small resolutions, to obtain the other small resolution we should consider $\Lambda^{-}$.)

It was proved in [15] that, up to homotheties and rescaling, there is a unique $\mathrm{SO}(4)-$ invariant symplectic form on the twistor space $R$ of $\mathbb{R}^{4}$ with infinite volume and whose sign changes under the antipodal map. From this twistorial view-point, it comes naturally from hyperbolic geometry. The Levi-Civita connection of the hyperbolic metric on $\mathbb{R}^{4} \cong H^{4}$ induces a metric connection in the vertical tangent bundle $V \rightarrow R$. 
Its curvature $-2 \pi i \omega$ determines the symplectic form. It follows from this that there is a symplectic action of the hyperbolic isometry group $\mathrm{SO}(4,1)$ extending that of $\mathrm{SO}(4)$.

In [9], Davidov-Muškarov-Grantcharov compute the total Chern form of the tangent bundle of the twistor space. Combining their calculation with the symplectomorphism of the twistor space and the small resolution in [15] gives a proof of Proposition 4. We give a separate proof later, using an alternative description of $R$.

2.3.2 Quaternionic description The next description, involving quaternions, is analogous to the standard picture of the twistor fibration $\mathbb{C P}^{3} \rightarrow S^{4}$ which we recall first. This description of the twistor space of $S^{4}$ appears in the original article of Atiyah-Hitchin-Singer [3] as well as paragraph 13.84 of Besse [4]. The analogous description of the twistor space of $H^{4}$ appears very briefly in the final line of 13.84 in [4] and more detail can be found in, for example, LeBrun [25; 26].

In the case of $S^{4}$, we begin by identifying $\mathbb{C}^{4} \cong \mathbb{H}^{2}$. Each complex line in $\mathbb{C}^{4}$ determines a quaternionic line in $\mathbb{H}^{2}$, giving a map $t: \mathbb{C P}^{3} \rightarrow \mathbb{H}^{1} \cong S^{4}$. The fibre $t^{-1}(p)$ is the $\mathbb{C P}^{1}$ of all complex lines in the quaternionic line $p$. A choice of positive-definite Hermitian form on $\mathbb{C}^{4}$ gives a Fubini-Study metric on $\mathbb{C P}^{3}$ and a round metric on $S^{4}$. The isometries $\mathrm{SO}(5)$ of $S^{4}$ are identified with those isometries of $\mathbb{C P}^{3}$ which preserve the fibres of $t$, giving an injection $\mathrm{SO}(5) \rightarrow \operatorname{PSU}(4)$.

To describe the twistor fibration of $H^{4}$ in an analogous way we use an indefinite Hermitian form: let $\mathbb{C}^{2,2}$ denote $\mathbb{C}^{4}$ together with the Hermitian form $h(w)=\left|w_{1}\right|^{2}+$ $\left|w_{2}\right|^{2}-\left|w_{3}\right|^{2}-\left|w_{4}\right|^{2}$ and consider the space

$$
N=\left\{w \in \mathbb{C}^{2,2}: h(w)<0\right\} / \mathbb{C}^{*}
$$

of negative lines in $\mathbb{C}^{2,2} ; N$ is an open set in $\mathbb{C P}^{3}$. Transverse to a negative line in $\mathbb{C}^{2,2}, h$ is indefinite with signature $(2,1)$; accordingly $N$ inherits a pseudo-Kähler metric of signature $(2,1)$, in the same way that a positive definite Hermitian form determines a Fubini-Study metric on $\mathbb{C P}^{3}$. The pseudo-Kähler metric makes $N$ into a symplectic manifold. Alternatively, one can see the $N$ as the symplectic reduction of $\mathbb{C}^{2,2}$ by the diagonal circle action; the Hamiltonian for this action is just $h$ and the reduction at $h=-1$ is $N$.

It is standard that $N$ is symplectomorphic to $R$. (One way to prove this is to use the fact that both admit Hamiltonian $T^{3}$-actions with equivalent moment polytopes.) To use this picture to relate $R$ to $H^{4}$, let $\mathbb{H}^{1,1}$ denote $\mathbb{H}^{2}$ together with the indefinite form $h^{\prime}(p)=\left|p_{1}\right|^{2}-\left|p_{2}\right|^{2}$. The map

$$
\left(w_{1}, w_{2}, w_{3}, w_{4}\right) \mapsto\left(w_{1}+j w_{2}, w_{3}+j w_{4}\right)
$$


identifies the Hermitian spaces $\mathbb{C}^{2,2} \cong \mathbb{H}^{1,1}$. There is a natural projection from $N$ to the space of negative quaternionic lines in $\mathbb{H}^{1,1}$, ie, to the quaternionic-hyperbolic space

$$
H_{\mathbb{H}}^{1}=\left\{p \in \mathbb{H}^{1,1}: h^{\prime}(p)<0\right\} / \mathbb{H}^{*} .
$$

In general, the space of negative lines in $\mathbb{H}^{n, 1}$ is the quaternionic analogue of hyperbolic or complex-hyperbolic $n$-space. When $n=1$, however, this is isometric to $H^{4}$, fourdimensional real-hyperbolic space. (In the lowest dimension, the symmetric spaces associated to complex or quaternionic geometry coincide with their equidimensional real analogues.)

So, analogous to $t: \mathbb{C P}^{3} \rightarrow S^{4}$, we have a projection $t: R \rightarrow H^{4}$; the fibre $t^{-1}(p)$ is the $\mathbb{C P}^{1}$ of all complex lines in the quaternionic line $p$. The pseudo-Kähler isometries of $R$ are $\operatorname{PSU}(2,2)$ whilst the isometries $\mathrm{SO}(4,1)$ of $H^{4}$ can be identified with those isometries of $R$ which preserve the fibres of $t$, giving an injection $\mathrm{SO}(4,1) \rightarrow \mathrm{PSU}(2,2)$. In this way we see again an action of $\mathrm{SO}(4,1)$ on $R$ by symplectomorphisms.

2.3.3 A coadjoint description Let $G$ be a Lie group and $\xi \in \mathfrak{g}^{*}$; denote the orbit of $\xi$ under the coadjoint action by $\mathcal{O}(\xi)$. It is a standard fact that there is a $G$-invariant symplectic structure on $\mathcal{O}(\xi)$. We will show how the small resolution fits into this general theory as a certain coadjoint orbit of $\mathrm{SO}(4,1)$.

The Lie algebra $\mathfrak{s o}(4,1)$ is $5 \times 5$ matrices of the form

$$
\left(\begin{array}{ll}
0 & u^{t} \\
u & A
\end{array}\right)
$$

where $u$ is a column vector in $\mathbb{R}^{4}$ and $A \in \mathfrak{s o}(4)$. Those elements with $u=0$ generate $\mathrm{SO}(4) \subset \mathrm{SO}(4,1)$.

The Killing form is nondegenerate on $\mathfrak{s o}(4,1)$ and so gives an equivariant isomorphism $\mathfrak{s o}(4,1) \cong \mathfrak{s o}(4,1)^{*}$. We consider the orbit of

$$
\xi=\left(\begin{array}{ll}
0 & 0 \\
0 & J_{0}
\end{array}\right)
$$

where $J_{0} \in \mathfrak{s o}(4)$ is a choice of almost complex structure on $\mathbb{R}^{4}$ (ie, $J_{0}^{2}=-1$ ). The subalgebra $\mathfrak{h}$ of matrices commuting with $\xi$ is those with $u=0$ and $\left[A, J_{0}\right]=0$, ie, $\mathfrak{h}=\mathfrak{u}(2) \subset \mathfrak{s o}(4) \subset \mathfrak{s o}(4,1)$. It follows that the stabiliser of $\xi$ is $\mathrm{U}(2)$ and so $\mathcal{O}(\xi) \cong \mathrm{SO}(4,1) / \mathrm{U}(2)$.

Lemma 5 There is an isomorphism of $\mathrm{U}(2)$-representation spaces:

$$
\mathfrak{s o}(4,1) \cong \mathfrak{u}(2) \oplus \Lambda^{2}\left(\mathbb{C}^{2}\right)^{*} \oplus \mathbb{C}^{2} .
$$


Proof There is a $U(2)$-equivariant isomorphism $\mathfrak{s o}(4) \cong \mathfrak{u}(2) \oplus \Lambda^{2}\left(\mathbb{C}^{2}\right)^{*}$. To see this, write $\mathfrak{s o}(4) \cong \Lambda^{2}\left(\mathbb{R}^{4}\right)^{*}$. Given a choice of almost complex structure on $\mathbb{R}^{4}$, any real 2-form $a$ can be written uniquely as $a=\alpha+\beta+\bar{\beta}$ where $\alpha \in \Lambda_{\mathbb{R}}^{1,1}$ is a real $(1,1)$-form and $\beta \in \Lambda^{2,0}$. Identifying $a$ with $(\alpha, \beta)$ gives a $\mathrm{U}(2)$-equivariant decomposition $\Lambda_{\mathbb{R}}^{2} \cong \Lambda_{\mathbb{R}}^{1,1} \oplus \Lambda^{2,0}$. But, via the Hermitian form, $\Lambda_{\mathbb{R}}^{1,1}$ is identified with skew-Hermitian matrices $\mathfrak{u}(2)$ and this gives the claimed isomorphism.

There is also an $\mathrm{SO}(4)$-equivariant isomorphism $\mathfrak{s o}(4,1) \cong \mathfrak{s o}(4) \oplus \mathbb{R}^{4}$. In the form (3), the $\mathfrak{s o}(4)$ summand is given by $u=0$ whilst the $\mathbb{R}^{4}$ summand by $A=0$. Combining these two isomorphisms completes the proof.

Lemma $6 U$ to scale, there is a unique $\mathrm{SO}(4,1)$-invariant symplectic form on $\mathcal{O}(\xi)=\mathrm{SO}(4,1) / \mathrm{U}(2)$.

Proof The existence follows from coadjoint orbit description. For uniqueness, we begin by describing all invariant nondegenerate 2 -forms. This amounts to describing all nondegenerate 2 -forms at a point which are invariant under the stabiliser $\mathrm{U}(2)$. From Lemma 5 the tangent space at a point is isomorphic as a $\mathrm{U}(2)$-representation space to $\Lambda^{2}\left(\mathbb{C}^{2}\right)^{*} \oplus \mathbb{C}^{2}$. Up to scale, there is a unique invariant 2 -form on each summand, giving two $\mathrm{SO}(4,1)$-invariant 2 -forms $a, b$ on $\mathrm{SO}(4,1) / \mathrm{U}(2)$. It follows that, up to scale, all nondegenerate invariant 2 -forms have the form $a+t b$ for $t \neq 0$. At most one of these can be closed, $t$ being fixed by the requirement that $\mathrm{d} a=-t \mathrm{~d} b$.

Corollary $7 \mathcal{O}(\xi)$ is symplectomorphic to $R$.

Proof This follows from the previous lemma and the transitive action of $\operatorname{SO}(4,1)$ on $R$ which can be seen in either the twistorial or quaternionic pictures.

We make a small digression to point out a simple consequence of the coadjoint orbit description of $R$ which is, perhaps, less apparent from its Kähler description as the total space of $\mathcal{O}(-1) \oplus \mathcal{O}(-1)$. We omit the details since this description of $R$ is not relevant to what follows.

Proposition $8 R$ is symplectomorphic to $T^{*}\left(S^{2} \times \mathbb{R}\right)$ with the symplectic structure $\omega_{\text {can }}+p^{*} \omega_{S^{2}}$, where $\omega_{\text {can }}$ is the canonical symplectic form on the cotangent bundle, $\omega_{S^{2}}$ is an area-one $2-$ form on $S^{2}$ and $p: T^{*}(S \times \mathbb{R}) \rightarrow S^{2}$ is the composition of projections $T^{*}\left(S^{2} \times \mathbb{R}\right) \rightarrow S^{2} \times \mathbb{R} \rightarrow S^{2}$.

Sketch proof As $R$ is a coadjoint orbit of $\mathrm{SO}(4,1)$, the action of $\mathrm{SO}(4,1)$ is Hamiltonian. It follows that $R$ admits a free Hamiltonian $\mathbb{R}^{3}$-action given as follows: fix 
a point $p$ at infinity on $H^{4}$ and consider the subgroup $\mathbb{R}^{3} \subset \mathrm{SO}(4,1)$ which acts by linear translations on the horospheres centred at $p$. The action on $R$ admits a section (ie, a smoothly varying choice of representative from each orbit) given by considering the restriction of $R \rightarrow H^{4}$ to a geodesic through $p$; this shows that the orbit space is $S^{2} \times \mathbb{R}$. Moreover, on this section, the symplectic form $\omega$ restricts to the pullback of an area-one form on $S^{2}$ via $S^{2} \times \mathbb{R} \rightarrow S^{2}$.

Now we appeal to a standard result concerning Hamiltonian $\mathbb{R}^{n}$ actions. Let $(M, \omega)$ be a symplectic $2 n$-manifold with a free Hamiltonian action of $\mathbb{R}^{n}$ and moment map $\mu: M \rightarrow\left(\mathbb{R}^{n}\right)^{*}$ with image $U$. Suppose there is a section $s: U \rightarrow M$ of $\mu$. Let $\alpha=s^{*} \omega$. Then translating the section via the $\mathbb{R}^{n}$ action gives a diffeomorphism $M \cong U \times \mathbb{R}^{n} \cong T^{*} U$ under which $\omega$ is identified with $\omega_{\text {can }}+\pi^{*} \alpha$. Here, $\pi: T^{U} \rightarrow U$. (If the section were Lagrangian, we would in the perhaps more familiar situation in which the moment map identifies $M$ symplectomorphically with the cotangent bundle of $U$.)

An alternative way to write this symplectic manifold is as the symplectic product $\mathcal{O}(-2) \times \mathbb{R}^{2}$. Here, $\mathcal{O}(-2)$ - the cotangent bundle to $S^{2}$-has the symplectic structure given by adding the pullbacks of the standard forms via the projection to $\mathbb{C P}^{1}$ and the resolution $\mathcal{O}(-2) \rightarrow \mathbb{C}^{2} / \mathbb{Z}_{2}$.

Corollary $9 R$ contains no Lagrangian 3-spheres.

Proof Since $\mathcal{O}(-2)$ is convex at infinity, this statement follows from recent work of Welschinger [44] (see, for example, Corollary 4.13).

2.3.4 An $\mathrm{SO}(4,1)$-invariant complex volume form We now give the proof of Proposition 4, which says that the small resolution admits an $\mathrm{SO}(4,1)$-invariant compatible almost complex structure and invariant complex volume form.

Proof of Proposition 4 We use the coadjoint orbit description. Lemma 5 says that at each point $z$ there is a $\mathrm{U}(2)$-equivariant isomorphism of the tangent space $T_{z} \cong$ $\Lambda^{2}\left(\mathbb{C}^{2}\right)^{*} \oplus \mathbb{C}^{2}$. Accordingly, there is a natural U(2)-invariant almost complex structure on $T_{z}$ which is $\omega$-compatible and, hence, an $\mathrm{SO}(4,1)$-invariant compatible almost complex structure $J$ on $\mathcal{O}(\xi)$.

For the complex volume form, note that $\mathrm{U}(2)$ acts trivially on $\Lambda^{3} T_{z}^{*} \cong \Lambda^{2}\left(\mathbb{C}^{2}\right) \otimes$ $\Lambda^{2}\left(\mathbb{C}^{2}\right)^{*}$ hence any nonzero element of $\Lambda^{3} T_{z}^{*}$ can be extended in a unique way to an $\mathrm{SO}(4,1)$-invariant complex volume form. 
We remark in passing that Lemma 5 and the almost complex structure used here also have a straightforward interpretation in the twistorial picture. Recall that $R \rightarrow H^{4}$ is a $\mathbb{C P}^{1}$-bundle and that $\omega$ is nondegenerate on the fibres. Hence $T R=V \oplus H$ where $V$ is the vertical tangent bundle and $H$ its complement with respect to $\omega$. This corresponds to the splitting in Lemma 5. The pseudo-Kähler metric is negative definite on $V$ and positive definite on $H$. Let

$$
J=-\left.J_{\text {int }}\right|_{V}+\left.J_{\text {int }}\right|_{H}
$$

where $J_{\text {int }}$ is the integrable complex structure on $R \subset \mathbb{C P}^{3} . J$ is an $\operatorname{SO}(4,1)$-invariant almost complex structure and coincides with that coming from the coadjoint orbit picture. It is an instance of the Eells-Salamon almost complex structure on the twistor space of a Riemannian four-manifold [12].

With this hyperbolic description in hand, we can use hyperbolic four-manifolds to produce symplectic quotients of the small resolution: a hyperbolic four-manifold carries a two-sphere bundle-its twistor space-whose total space is symplectic with trivial canonical bundle. Of course, any compact example will have infinite fundamental group. We will produce a simply connected example in Section 4 by considering a certain hyperbolic orbifold.

\section{Complex examples}

Compact complex manifolds will be built starting from hyperbolic orbifold metrics on $S^{3}$ with cone angle $2 \pi / m$ along a knot $K \subset S^{3}$. When such a metric exists, $K$ is said to be $2 \pi / m$-hyperbolic. Such knots are well-known to be plentiful. As is explained in [6], it is a consequence of Thurston's orbifold Dehn surgery theorem that when $K$ is a hyperbolic knot-ie, when $S^{3} \backslash K$ admits a complete finite-volume hyperbolic metric $-K$ is also $2 \pi / m$-hyperbolic for all $m \geq 3$ with one sole exception, namely when $K$ is the figure eight knot and $m=3$. There are also infinitely many $\pi$-hyperbolic knots. (We are grateful to Michel Boileau for advice on this matter.)

Let $H^{3} / \Gamma$ be a hyperbolic orbifold metric on $S^{3}$ with cone angle $2 \pi / m$ along a knot $K$ where $\Gamma \subset \operatorname{PSL}(2, \mathbb{C})$ is the orbifold fundamental group. It is standard that there is smooth hyperbolic manifold $M$ which is an $m$-fold cyclic cover $M \rightarrow S^{3}$ branched along the knot $K$. (By Mayer-Vietoris, $H_{1}\left(S^{3} \backslash K\right)=\mathbb{Z}$; hence there is a homomorphism $\pi_{1}\left(S^{3} \backslash K\right) \rightarrow \mathbb{Z}$ which in turn induces a homomorphism $\psi: \Gamma \rightarrow \mathbb{Z}_{m}$; the kernel $\Gamma^{\prime}$ of $\psi$ has no fixed points on $H^{3}$ and is the fundamental group of $M$.)

Since $M$ is a hyperbolic manifold, its spin bundles are complex manifolds with trivial canonical bundle (as is described in Section 2.2). When the spin-structure $P \rightarrow M$ is 
well-chosen, the generator of the $\mathbb{Z}_{m}$-action lifts to $P$ where it generates a $\mathbb{Z}_{2 m}$-action. To produce a simply connected quotient of $P$, we then "twist" this action around the knot; this induces fixed points in the fibres over $K$ and results in a singular quotient for which all the fibres are simply connected, giving a simply connected total space. Finally, we obtain a smooth manifold by taking a crepant resolution.

\subsection{The model singularity and resolution}

We begin by considering the model situation. Take a geodesic $\gamma$ in $H^{3}$ and let $\mathbb{Z}_{m}$ act on $H^{3}$ by fixing $\gamma$ pointwise and rotating perpendicular to $\gamma$ by $2 \pi / \mathrm{m}$. Let $\varphi=e^{\pi i / m}$. In appropriate coordinates, $\mathbb{Z}_{m} \subset \operatorname{PSL}(2, \mathbb{C})$ is generated by the class $[U]$ of the diagonal matrix $U$ with entries $\varphi, \varphi^{-1}$.

The group $\mathbb{Z}_{m} \in \operatorname{PSL}(2, \mathbb{C})$ is covered by the copy of $\mathbb{Z}_{2 m} \subset \operatorname{SL}(2, \mathbb{C})$ generated by $U$. The action of $\mathbb{Z}_{m}$ on $H^{3}$ is covered by the action of $\mathbb{Z}_{2 m}$ by right multiplication on $\operatorname{SL}(2, \mathbb{C})$. However, this action on $\operatorname{SL}(2, \mathbb{C})$ is free and so the quotient has nontrivial fundamental group. To produce a singular but simply connected quotient, we "twist" to introduce fixed points. This additional twist is given by simultaneously multiplying on the left.

Consider the action of $\mathbb{Z}_{m}$ on $\operatorname{SL}(2, \mathbb{C})$ generated by conjugation $A \mapsto U^{-1} A U$. Explicitly, in coordinates, the generator is

$$
\left(\begin{array}{cc}
x & y \\
z & w
\end{array}\right) \mapsto\left(\begin{array}{cc}
x & \varphi^{-2} y \\
\varphi^{2} z & w
\end{array}\right) .
$$

The points of $\operatorname{SL}(2, \mathbb{C})$ fixed by $\mathbb{Z}_{m}$ are the $\mathbb{C}^{*}$ subgroup of diagonal matrices. On a tangent plane normal to the fixed points, the $\mathbb{Z}_{m}$-action is that of the $A_{m}$-singularity (ie, $\mathbb{C}^{2} / \mathbb{Z}_{m}$ with action generated by $\left.U^{2} \in \mathrm{SU}(2)\right)$. Left multiplication on $\operatorname{SL}(2, \mathbb{C})$ by the $\mathbb{C}^{*}$ subgroup of diagonal matrices is free and commutes with the action of $\mathbb{Z}_{m}$; hence it descends to a free $\mathbb{C}^{*}$-action on the quotient. The $\mathbb{C}^{*}$-action is transitive on the fixed locus and so gives an identification of the neighbourhood of the orbifold points with the product of $\mathbb{C}^{*} \times V$, where $V$ is a neighbourhood of the singular point in the $A_{m}$-singularity.

The $\mathbb{Z}_{m}$-action preserves the fibres of $\operatorname{SL}(2, \mathbb{C}) \rightarrow H^{3}$ and covers the $\mathbb{Z}_{m}$ action on $H^{3}$ - the fibres are right cosets of $\mathrm{SU}(2)$ and $U \in \mathrm{SU}(2)$ - hence there is a projection $\mathrm{SL}(2, \mathbb{C}) / \mathbb{Z}_{m} \rightarrow H^{3} / \mathbb{Z}_{m}$. The $\mathbb{Z}_{m}$-fixed points form a circle bundle over the geodesic $\gamma$. We have implicitly oriented $\gamma$ in our choice of coordinates (4); the image of the fixed points in the frame bundle $\operatorname{PSL}(2, \mathbb{C})$ are those frames whose first vector is positively tangent to $\gamma$. 
Since the $A_{m}$-singularity admits a crepant resolution, it follows that $\operatorname{SL}(2, \mathbb{C}) / \mathbb{Z}_{m}$ does too. The exceptional divisor of the resolution $W \rightarrow \operatorname{SL}(2, \mathbb{C}) / \mathbb{Z}_{m}$ maps to the $\mathbb{C}^{*}$ of singular points with fibre a chain of $m-1$ rational curves over each point, as in the standard crepant resolution of the $A_{m}$ singularity.

In our model example, the $\mathbb{Z}_{m}$ fixed locus in $H^{3}$ is a geodesic $\gamma$. In our compact examples, the branch locus will be a closed geodesic loop. The geodesic $\gamma$ is "closed up" by the action of $\mathbb{Z}$ on $\operatorname{SL}(2, \mathbb{C})$ generated by right-multiplication by the diagonal matrix with entries $a, a^{-1}$, where $a \in \mathbb{C}$ has $|a|>1$. This action is free and commutes with the $\mathbb{Z}_{m}$ action described above, hence it induces an action of $\mathbb{Z}$ on $\operatorname{SL}(2, \mathbb{C}) / \mathbb{Z}_{m}$ and on the crepant resolution $W$. The quotient $W / \mathbb{Z}$ is a crepant resolution of $\operatorname{SL}(2, \mathbb{C}) /\left(\mathbb{Z}_{m} \oplus \mathbb{Z}\right)$, a complex orbifold whose singular locus is an elliptic curve. This is the singularity which will actually appear in our compact examples.

Finally note that the free $\mathbb{C}^{*}$-action on $\operatorname{SL}(2, \mathbb{C}) / \mathbb{Z}_{m}$ described above commutes with the action of $\mathbb{Z}$ and so descends to $\operatorname{SL}(2, \mathbb{C}) /\left(\mathbb{Z}_{m} \oplus \mathbb{Z}\right)$. It induces a locally free $\mathbb{C}^{*}$-action on the resolution, ie, it is generated by a nowhere vanishing vector field. This is a feature which will be shared by our compact examples.

\subsection{Compact examples}

We now consider a hyperbolic orbifold metric on $S^{3}$ with cone angle $2 \pi / \mathrm{m}$ along a knot $K$. It is a standard fact that there a is smooth hyperbolic manifold $M$ which is an $m$-fold cyclic cover $M \rightarrow S^{3}$ branched along the knot $K . \mathbb{Z}_{m}$ acts by isometries on $M$, fixing the geodesic branch locus pointwise and rotating the normal bundle by $2 \pi / m$. The action of $\mathbb{Z}_{m}$ on the universal cover $H^{3}$ of $M$ is precisely the situation considered in Section 3.1: $\mathbb{Z}_{m} \subset \operatorname{PSL}(2, \mathbb{C}$ ) is generated (in appropriate coordinates) by the class $[U]$ of a matrix $U$ which is diagonal with entries $\varphi$ and $\varphi^{-1}$, where $\varphi=e^{\pi i / m}$.

In order to produce a complex orbifold we first try to lift the $\mathbb{Z}_{m}$-action to a spin bundle of $M$. Let $Q$ denote the frame bundle of $M$ and $P$ a choice of spin bundle. The generator of the $\mathbb{Z}_{m}$-action on $M$ induces a diffeomorphism $f$ of $Q$ and we aim to choose the spin structure so that $f$ lifts to $P$. For this we state the following standard result:

Lemma 10 Let $Y$ be a connected manifold, $f: Y \rightarrow Y$ a diffeomorphism and $Y_{h} \rightarrow Y$ the double cover corresponding to the element $h \in H^{1}\left(Y, \mathbb{Z}_{2}\right)$. Then $f$ lifts to $Y_{h}$ if and only if $f^{*} h=h$.

Double covers of $Q$ are parametrised by $H^{1}\left(Q, \mathbb{Z}_{2}\right)$ and spin structures correspond to elements which are nonzero on restriction to the fibres of $Q \rightarrow M$. So to lift the 
generator $f$ of the $\mathbb{Z}_{m}$-action to $P$ we must find a suitable invariant $h \in H^{1}\left(Q, \mathbb{Z}_{2}\right)$. For certain values of $m$, this can always be done.

Lemma 11 If $m$ is odd or $m=2^{r}$ then there is a $\mathbb{Z}_{m}$-invariant element of $H^{1}\left(Q, \mathbb{Z}_{2}\right)$ corresponding to a spin structure $P \rightarrow Q$.

Proof If $m$ is odd, take any $h \in H^{1}\left(Q, \mathbb{Z}_{2}\right)$ whose restriction to a fibre is nonzero and average over the group $\mathbb{Z}_{m}$ to give an invariant element. Since $m$ is odd, the fibrewise restriction remains nonzero.

When $m=2^{r}$ we use a lemma from knot theory: the $2^{r}$-fold cover $M^{\prime} \rightarrow S^{3}$ branched along a knot has $H^{1}\left(M, \mathbb{Z}_{2}\right)=0$ (see, eg, page 16 of Gordon [18]). It follows that $M$ has a unique spin structure, $H^{1}\left(Q, \mathbb{Z}_{2}\right) \cong \mathbb{Z}_{2}$ and the generator of $H^{1}\left(Q, \mathbb{Z}_{2}\right)$ is $\mathbb{Z}_{m}$-invariant.

So, when $m$ is odd or $m=2^{r}$, there is a choice of spin structure $P \rightarrow Q$ for which generator of the $\mathbb{Z}_{m}$-action lifts. Upstairs in $P$, the lifted action has order $2 m$. This can be seen by considering the $\mathbb{Z}_{m}$-action on a fibre of $Q$ over a point in $K$ : here we have lifted the standard action of rotation by $2 \pi / m$ on $\operatorname{SO}(3)$ to its double cover $\mathrm{SU}(2)$; it is straightforward to check the order upstairs is $2 m$. The action of $\mathbb{Z}_{2 m}$ on the universal cover $\mathrm{SL}(2, \mathbb{C})$ of $P$ is generated (again, in appropriate coordinates) by right multiplication by the matrix $U$ which is diagonal with entries $\varphi$ and $\varphi^{-1}$, where $\varphi=e^{\pi i / m}$.

Now we can "twist" the action around the knot, to introduce a singularity just as in the model case. We define an action of $\mathbb{Z}_{m}$ on $P$ by sending the generator to conjugation by $U$. More correctly, this defines an action of $\mathbb{Z}_{m}$ on the universal cover of $P$; but we have only altered the original $\mathbb{Z}_{2 m}$-action by left-multiplication and all deck transformations come from right-multiplication, so the $\mathbb{Z}_{m}$-action on $\operatorname{SL}(2, \mathbb{C})$ commutes with the action of $\pi_{1}(M)$ by deck transformations and hence descends to a $\mathbb{Z}_{m}$-action on $P$. We consider the quotient $P / \mathbb{Z}_{m}$. Just as the model singularity projects $\operatorname{SL}(2, \mathbb{C}) / \mathbb{Z}_{m} \rightarrow H^{3} / \mathbb{Z}_{m}$ there is a projection $P / \mathbb{Z}_{m} \rightarrow S^{3}$. Away from the knot $K$ this is a locally trivial $S^{3}$-bundle, over a small neighbourhood of $K$ we have precisely the model singularity considered above in Section 3.1. It follows that we can glue in a neighbourhood of the exceptional divisor from the model crepant resolution to obtain a crepant resolution $X \rightarrow P / \mathbb{Z}_{m}$, giving a complex manifold with trivial canonical bundle.

Lemma 12 The resolution $X$ admits a unique (up to scale) nowhere-vanishing holomorphic vector field. Moreover, it generates a $\mathbb{C}^{*}$-action. 
Proof We start by showing the existence of the vector field. The $\mathbb{Z}_{m}$-action on $P$ is given (on the universal cover $\operatorname{SL}(2, \mathbb{C})$ ) by conjugation by a diagonal matrix with determinant 1 . Hence, on the universal cover, it commutes with left-multiplication by the whole $\mathbb{C}^{*}$ of such diagonal matrices. Since the remaining deck transformations are all given by right-multiplication, they also commute with left-multiplication by $\mathbb{C}^{*}$, hence this $\mathbb{C}^{*}$-action descends to $P / \mathbb{Z}_{m}$ and then lifts to the resolution $X$. The action is locally free on $X$-ie, it is generated by a nowhere vanishing vector field-since the same is true of the $\mathbb{C}^{*}$-action on $\operatorname{SL}(2, \mathbb{C})$.

Next we show uniqueness. Notice first that every holomorphic vector field $v$ on $X$ defines a holomorphic vector field on $P$. Indeed $v$ defines a holomorphic vector field on the complement to the singular curve of $P / \mathbb{Z}_{m}$, and so it can be lifted to the complement of the branch locus of the $\mathbb{Z}_{m}$ action on $P$. This last locus is a union of curves, so by Hartog's extension theorem (see, eg, Griffiths and Harris [19]) the lifted field extends holomorphically to $P$. Finally, recall that the tangent bundle of $P$ is trivial; by construction there is a unique vector field on $P$ invariant under the action of $\mathbb{Z}_{m}$.

\subsection{Simple connectivity and non-Kählerity}

To show that our examples are simply connected, we begin by with a result from algebraic topology. We recall that a space is said to be $L C^{n}$ if every point has a neighbourhood which is $n$-connected (ie, all homotopy groups up to and including $\pi_{n}$ are trivial). In particular, all $\mathrm{CW}$-complexes are $L C^{n}$ for every $n$, which is the most generality we will consider. Moreover, we need only the $n=2$ case of the following result.

Theorem 13 (Smale [38]) If $f: X \rightarrow Y$ is a proper, surjective map of connected, locally compact, separable metric spaces, $X$ is $L C^{n}$, and each fibre is $L C^{n-1}$ and $(n-1)$-connected, then the induced homomorphism $\pi_{j}(X) \rightarrow \pi_{j}(Y)$ is an isomorphism for $j \leq n-1$ and surjective for $j=n$.

Lemma 14 Let $X$ be the complex threefold associated to a $2 \pi / m$-hyperbolic knot, as described above. Then $X$ is simply connected.

Proof First, we consider the projection $f: P / \mathbb{Z}_{m} \rightarrow S^{3}$. We will prove that the group $\pi_{1}\left(P / \mathbb{Z}_{m}\right)$ is trivial. Away from the knot $K$ this is a locally trivial $S^{3}$-fibration. The fibre of $f$ over a point in $K$ is the quotient $\mathrm{SU}(2) / \mathbb{Z}_{m}$ via the action (4) which is easily seen to be homeomorphic to $S^{3}$. Hence Theorem 13 applies to $f$, giving $\pi_{1}\left(P / \mathbb{Z}_{m}\right) \cong \pi_{1}\left(S^{3}\right)=1$. 
To deduce that the resolution $X$ is also simply connected we apply the $n=2$ case of Theorem 13 again, this time to the map $r: X \rightarrow P / \mathbb{Z}_{m}$. Away from the singular locus, $r$ is one-to-one; meanwhile each point on the singular locus has preimage a chain of $m-1$ copies of $S^{2}$. Since all fibres are simply connected and locally simply connected, the result follows.

Lemma 15 There is no compatible Kähler structure on the complex threefold associated to a $2 \pi / m$-hyperbolic knot.

Proof The fact that our examples are simply connected (Lemma 14) and admit a locally free $\mathbb{C}^{*}$-action (Lemma 12) implies that they have no compatible Kähler metric. For if they admitted a compatible Kähler metric, by averaging it would be possible to find an $S^{1} \subset \mathbb{C}^{*}$ invariant Kähler form. Now $b_{1}=0$ implies that the symplectic $S^{1}$-action would, in fact, be Hamiltonian and hence have fixed points.

\subsection{Diffeomorphism type for $\pi$-hyperbolic knots}

We now turn to the question of the diffeomorphism type of our examples in the topologically most simple case, that of a $\pi$-hyperbolic knot. We proceed via Wall's classification theorem [42]. Wall's result states that oriented, smooth, simply connected, spin, 6-manifolds with torsion-free cohomology are determined up to oriented-diffeomorphism by:

- the integer $b_{3}$,

- the symmetric trilinear map $H^{2} \times H^{2} \times H^{2} \rightarrow \mathbb{Z}$ given by cup product,

- the homomorphism $H^{2} \rightarrow \mathbb{Z}$ given by cup product with the first Pontrjagin class.

The goal of this section is to prove:

Theorem 16 Given a $\pi$-hyperbolic knot, the resulting complex threefold constructed above is diffeomorphic to $2\left(S^{3} \times S^{3}\right) \#\left(S^{2} \times S^{4}\right)$.

To compute the cohomology of the complex threefold, we begin with the topology of the orbifold $P / \mathbb{Z}_{2}$. In fact, the results here hold for $P / \mathbb{Z}_{m}$ with any choice of $m$.

Let $K \subset S^{3}$ be a $2 \pi / m$-hyperbolic knot (with $m$ odd or $m=2^{r}$ ) and let $U$ be a small tubular neighbourhood of $K$. We write the boundary of $U$ as $S_{1}^{1} \times S_{2}^{1}$ where $S_{1}^{1}$ is a meridian circle, which is contractible in $U$, and $S_{2}^{1}$ is a longitudinal circle, which 
is contractible in $S^{3} \backslash K$. Let $f: P / \mathbb{Z}_{m} \rightarrow S^{3}$ denote the projection from the complex orbifold to the hyperbolic orbifold. Write $X_{1}=f^{-1}\left(S^{3} \backslash K\right)$ and $X_{2}=f^{-1}(U)$. In what follows, (co)homology groups are taken with coefficients in $\mathbb{Z}$ unless explicitly stated. We begin with a couple of standard topological lemmas.

Lemma 17 Given any knot $K \subset S^{3}$, the knot complement has $H_{1}\left(S^{3} \backslash K\right) \cong \mathbb{Z}$, generated by the class of $S_{1}^{1}$, whilst $H_{2}\left(S^{3} \backslash K\right) \cong 0 \cong H_{3}\left(S^{3} \backslash K\right)$.

Lemma 18 Every $\mathrm{SO}(4)$-bundle over a 3-manifold with $H_{2} \cong 0 \cong H_{3}$ is trivial.

We apply these results to show that for $j=1,2$, the subset $f^{-1}\left(S_{j}^{1}\right)$ carries all the homology of $X_{j}$.

Lemma $19 f: X_{1} \rightarrow S^{3} \backslash K$ is a trivial $S^{3}$-fibration. The inclusion $f^{-1}\left(S_{1}^{1}\right) \rightarrow X_{1}$ induces an isomorphism on homology.

Proof $\operatorname{SL}(2, \mathbb{C}) \rightarrow H^{3}$ is an $\mathrm{SU}(2)$-bundle so, in particular is an $S^{3}$-bundle with structure group $\mathrm{SO}(4)$. Moreover, this $\mathrm{SO}(4)$ structure is preserved by the image of $\mathrm{SU}(2) \times \mathrm{SL}(2, \mathbb{C})$ in $\mathrm{SO}(4, \mathbb{C})$. Accordingly, away from $K$, the map $f: X_{1} \rightarrow S^{3} \backslash K$ has structure group $\mathrm{SO}(4)$. The result now follows from the previous two lemmas.

Lemma $20 X_{2}$ retracts to $f^{-1}(K)$. The inclusion $f^{-1}\left(S_{2}^{1}\right) \rightarrow X_{2}$ induces an isomorphism on homology.

Proof To prove that $X_{2}$ retracts as claimed it suffices to consider the model of Section 3.1, SL(2, $\mathbb{C}) /\left(\mathbb{Z} \oplus \mathbb{Z}_{m}\right)$. The orthogonal retraction of $H^{3}$ onto the $\left(\mathbb{Z} \oplus \mathbb{Z}_{m}\right)$-fixed geodesic induces a retraction of a small tubular neighbourhood of the geodesic loop in the quotient $H^{3} /\left(\mathbb{Z} \oplus \mathbb{Z}_{m}\right)$. This lifts to give the claimed retraction of the preimage of the tubular neighbourhood in $\operatorname{SL}(2, \mathbb{C}) /\left(\mathbb{Z} \oplus \mathbb{Z}_{m}\right)$. It follows from this that the embedding $f^{-1}\left(S_{2}^{1}\right) \rightarrow X_{2}$ induces an isomorphism on homology.

Now we are in a position to compute the homology of the complex orbifold.

Proposition $21 P / \mathbb{Z}_{m}$ has the integral homology of $S^{3} \times S^{3}$.

Proof We compute the cohomology of $P / \mathbb{Z}_{m}$ by applying the Mayer-Vietoris sequence to the pair $\left(X_{1}, X_{2}\right)$. For this we need the maps $\left(i_{1}\right)_{*}$ and $\left(i_{2}\right)_{*}$ induced on homology by the inclusions $i_{1}: X_{1} \cap X_{2} \rightarrow X_{1}$ and $i_{2}: X_{1} \cap X_{2} \rightarrow X_{2}$. It follows from Lemma 19 that $X_{1} \cap X_{2}$ is the product $f^{-1}(U \backslash K) \times S^{3}$. In particular, it retracts to $f^{-1}\left(S_{1}^{1} \times S_{2}^{1}\right)=S_{1}^{1} \times S_{2}^{1} \times S^{3}$. 
Let $i_{1}^{\prime}$ and $i_{2}^{\prime}$ denote the compositions of the retraction of $X_{1} \cap X_{2}$ to $S_{1}^{1} \times S_{2}^{1} \times S^{3}$ with a consecutive projection to $S_{1}^{1} \times S^{3}$ and $S_{2}^{1} \times S^{3}$ respectively. The preceding Lemma 19 and Lemma 20 show that the images of the maps $\left(i_{1}\right)_{*}$ and $\left(i_{2}\right)_{*}$ coincide with the images of the maps $\left(i_{1}^{\prime}\right)_{*}$ and $\left(i_{2}^{\prime}\right)_{*}$ respectively. It follows from this that the Mayer-Vietoris sequence of the pair $\left(X_{1}, X_{2}\right)$ can be identified with the MayerVietoris sequence of the pair $\left(\left(S^{3} \backslash K\right) \times S^{3}, U \times S^{3}\right)$. Since this second sequence calculates the homology of $S^{3} \times S^{3}$, the proposition is proved.

The singular locus of $P / \mathbb{Z}_{m}$ is an elliptic curve $C$. The next step is to compute the homology of the pair $\left(P / \mathbb{Z}_{m}, C\right)$.

Lemma 22 The nonzero relative homology groups $H_{j}\left(P / \mathbb{Z}_{m}, C\right)$ are

$$
H_{2}\left(P / \mathbb{Z}_{m}, C\right) \cong \mathbb{Z}^{2}, \quad H_{3}\left(P / \mathbb{Z}_{m}, C\right) \cong \mathbb{Z}^{3}, \quad H_{6}\left(P / \mathbb{Z}_{m}, C\right) \cong \mathbb{Z} .
$$

Proof This follows immediately from the exact sequence of the pair $\left(P / \mathbb{Z}_{m}, C\right)$ along with the fact that $P / \mathbb{Z}_{m}$ has the integral homology of $S^{3} \times S^{3}$.

To convert this into information about the homology of the resolution $X$ we restrict to the case when $K$ is $\pi$-hyperbolic.

Proposition 23 Let $X$ be the complex threefold constructed from a $\pi$-hyperbolic knot, as described above. Then $X$ has the integral homology of $2\left(S^{3} \times S^{3}\right) \#\left(S^{2} \times S^{4}\right)$.

Proof Let $E \subset X$ denote the exceptional divisor of the resolution $X \rightarrow P / \mathbb{Z}_{2}$. The singularity in $P / \mathbb{Z}_{2}$ is locally the product of the elliptic curve $C$ with the $A_{2}$-singularity in $\mathbb{C}^{2}$. So $E \cong \mathbb{C P}^{1} \times C$, with normal bundle $\mathcal{O}(-2)$ pulled back from $\mathbb{C P}^{1}$.

Since $X / E$ (where $E$ is crushed to a point) is homeomorphic to $\left(P / \mathbb{Z}_{2}\right) / C$, it follows that the relative groups $H_{j}(X, E) \cong H_{j}\left(P / \mathbb{Z}_{2}, C\right)$ are given by Lemma 22 . We now consider the long exact sequence of the pair $(X, E)$. Since $H_{5}(E)=0=H_{5}(X, E)$, we have that $H_{5}(X)=0$. Meanwhile, since $H_{5}(X, E)=0=H_{4}(X, E)$ we have that $H_{4}(X) \cong H_{4}(E) \cong \mathbb{Z}$.

From here we can compute all the Betti numbers. Indeed, by Poincaré duality, $b_{2}(X)=$ $b_{4}(X)=1$; now considering the alternating sum of the ranks of the groups in the part of the sequence from $H_{4}(X, E)=0$ to $H_{1}(X)=0$ gives $b_{3}=4$. So the rational homology of $X$ coincides with that of the connected sum. It remains to show that $H_{2}(X)$ and $H_{3}(X)$ are torsion-free.

Since $H_{4}(X, E)=0$, the long exact sequence gives:

$$
0 \rightarrow H_{3}(E) \rightarrow H_{3}(X) \stackrel{j}{\rightarrow} H_{3}(X, E) \rightarrow \cdots
$$


As $H_{3}(X, E) \cong \mathbb{Z}^{3}$ is torsion-free, all torsion in $H_{3}(X)$ must be contained in ker $j$. However, $\operatorname{ker} j \cong H_{3}(E) \cong \mathbb{Z}^{2}$ is torsion-free, hence so is $H_{3}(X)$.

The argument for $H_{2}(X)$ is more involved. First, note that since $H_{1}(X)=0$ and $H_{2}(X, E) \cong \mathbb{Z}^{2} \cong H_{1}(E)$, the map $H_{2}(X, E) \rightarrow H_{1}(E)$ in the sequence of the pair $(X, E)$ is an isomorphism. Now the preceding part of the sequence gives that $H_{2}(E) \rightarrow H_{2}(X)$ is surjective. $E \cong C \times \mathbb{C P}^{1}$, so $H_{2}(E) \cong \mathbb{Z}^{2}$ is generated by the class of a $\mathbb{C P}^{1}$-fibre and the class of a $C$-fibre. Since $H_{2}(X)$ has rank 1 , to show that $H_{2}(X) \cong \mathbb{Z}$ it suffices to show that the $C$-fibre in $E$ is null-homologous in $X$.

For this it suffices to consider the model case of the resolution $X^{\prime} \rightarrow \operatorname{SL}(2, \mathbb{C}) /\left(\mathbb{Z}_{2} \oplus \mathbb{Z}\right)$ as in Section 3.1. This is because if there is a three-chain bounding $C$ in $X^{\prime}$, then the retraction in $H^{3}$ onto the fixed geodesic pushes this 3-chain into an arbitrarily small neighbourhood of the exceptional divisor; hence there is such a 3 -chain in the resolution $X$.

$\operatorname{SL}(2, \mathbb{C}) /\left(\mathbb{Z}_{2} \oplus \mathbb{Z}\right)$ is the quotient of $\operatorname{SL}(2, \mathbb{C}) / \mathbb{Z}$ by an involution with fixed locus an elliptic curve $C$. Away from $C$, there is a two-to-one map $\operatorname{SL}(2, \mathbb{C}) / \mathbb{Z} \rightarrow X^{\prime}$. This extends to the blow-up $\hat{X}$ of $\operatorname{SL}(2, \mathbb{C}) / \mathbb{Z}$ along $C$ to give a ramified double cover $\hat{X} \rightarrow X^{\prime}$. (This is analogous to the fact that, for bundles over $\mathbb{C P}^{1}$, the map $\mathcal{O}(-1) \rightarrow \mathcal{O}(-2)$ induced by $z \mapsto z^{2}$ in the fibres gives a ramified double cover of the $A_{2}$-resolution.) The ramification locus in $\hat{X}$ is the exceptional divisor $\hat{E}$ of the blow up $\hat{X} \rightarrow \operatorname{SL}(2, \mathbb{C}) / \mathbb{Z} . \hat{E}$ is identified with the branch locus in $X^{\prime}$, which is the exceptional divisor $E$ of the resolution $X^{\prime} \rightarrow \operatorname{SL}(2, \mathbb{C}) /\left(\mathbb{Z}_{2} \oplus \mathbb{Z}\right)$. So $\widehat{E} \cong \mathbb{C P}^{1} \times C$ and to show that a $C$-fibre of $E$ is null-homologous in $X^{\prime}$ it suffices to show that a $C$-fibre of $\widehat{E}$ is null-homologous in $\widehat{X}$.

To prove this statement, first note that $\operatorname{SL}(2, \mathbb{C}) / \mathbb{Z}$ is homeomorphic to $S^{3} \times S^{1} \times \mathbb{R}^{2}$. The elliptic curve $C$ corresponds to taking the product of a Hopf circle $S^{1} \subset S^{3}$ with $S^{1} \times\{\mathrm{pt}\}$. Choosing a different Hopf circle gives another copy $C^{\prime}$ of $C$ which lies entirely inside $(\operatorname{SL}(2, \mathbb{C}) / \mathbb{Z}) \backslash C$. On the one hand, $C^{\prime}$ is null-homologous in $(\mathrm{SL}(2, \mathbb{C}) / \mathbb{Z}) \backslash C$ (simply move it in the $\mathbb{R}^{2}$-direction so that it lies in a complete copy of $S^{3} \times S^{1}$ ). On the other hand, when thought of as a 2-cycle in the blow-up $\hat{X}$, $C^{\prime}$ is homologous to a $C$-fibre of $\widehat{E}$. Hence the $C$-fibre of $\widehat{E}$ is zero in homology and the proposition is proved.

Lemma 24 Let $X$ be the complex threefold constructed from a $\pi$-hyperbolic knot, as described above. Then all the Chern classes of $X$ vanish.

Proof We already know that $c_{1}=0$ and $c_{3}=0$ (as it is the Euler characteristic). Since $H_{4}(X)$ is generated by the exceptional divisor $E$, to prove $c_{2}=0$ it suffices to show 
that $\left\langle c_{2}, E\right\rangle=0$. Now, the normal bundle to $E \cong \mathbb{C P}^{1} \times C$ is $\mathcal{O}(-2)$ pulled back from $\mathbb{C P}^{1}$. Combining this with the fact that $c_{1}(X)=0$ gives that over $E$ we have a topological isomorphism $\left.T X\right|_{E} \cong \mathcal{O}(-2) \oplus \mathcal{O}(2) \oplus \mathcal{O}$. Since all of these bundles are pulled back to $E$ from $\mathbb{C P}^{1}$ we see that $\left\langle c_{2}, E\right\rangle=0$, as claimed.

We are now ready to prove that $X$ is diffeomorphic to $2\left(S^{3} \times S^{3}\right) \#\left(S^{2} \times S^{4}\right)$.

Proof of Theorem 16 In order to apply Wall's Theorem we must first check that $X$ is spin, which follows from the fact that it is complex with trivial canonical bundle.

Next, we must show the cup product is trivial on $H^{2}$. In the proof of Proposition 23, we saw that $H^{2}(X)$ is generated by the Poincare dual $e$ to the exceptional divisor $E$. Since $H_{4}(X)$ is generated by $E$, it suffices to check that $\left\langle e^{2}, E\right\rangle=0$. The normal bundle of $E \cong \mathbb{C P}^{1} \times C$ is pulled back from $\mathbb{C P}^{1}$. Hence, when restricted to $E$, $e$ is also pulled back from $\mathbb{C P}^{1}$ and so squares to zero.

Finally, we must show that $p_{1}(X)=0$, but this follows from the formula $p_{1}=c_{1}^{2}-2 c_{2}$ combined with $c_{1}=0=c_{2}$.

\subsection{Recovering the orbifold from the threefold}

In this section we prove that the hyperbolic orbifold can be recovered from the complex threefold, so distinct orbifolds lead to distinct threefolds.

Theorem 25 Let $X$ and $X^{\prime}$ be the complex threefolds constructed from hyperbolic orbifolds $N$ and $N^{\prime}$ as above. If $X$ and $X^{\prime}$ are biholomorphic then $N$ and $N^{\prime}$ are isometric.

Proof Suppose that $X$ is constructed from an orbifold metric on $S^{3}$ with cone angle $2 \pi / m$, whilst $X^{\prime}$ involves the cone angle $2 \pi / m^{\prime}$. Away from the elliptic curve $C \subset P / \mathbb{Z}_{m}$ of singular points, $r: X \rightarrow P / \mathbb{Z}_{m}$ is an isomorphism. The exceptional locus $E=r^{-1}(C)$ is biholomorphic to the product of $C$ with a chain $\Theta_{m}$ of $m-1$ copies of $\mathbb{C P}^{1}$ as in the $A_{m}$-resolution.

First, we claim that any analytic surface in $X$ is contained in $E$. Any surface not contained in $E$ would project to a surface in $P / \mathbb{Z}_{m}$; its preimage would then be an analytic surface in $P=\operatorname{SL}(2, \mathbb{C})$. However, it is known that the quotient of $\operatorname{SL}(2, \mathbb{C})$ by a cocompact subgroup never contains an analytic surface [23, Theorem 9.2]. It follows that $E$ is the union of all surfaces in $X$. Since $X$ and $X^{\prime}$ are biholomorphic, the union of all surfaces in $X^{\prime}$ is also biholomorphic to $C \times \Theta_{m}$. It follows that the exceptional loci $E^{\prime}, E$ are biholomorphic and so $m=m^{\prime}$. 
Next, note that a neighbourhood of $E$ is canonically biholomorphic to the product of $C$ with a neighbourhood of $\Theta_{m}$ in the $A_{m}$-resolution. The canonical biholomorphism is provided by the $\mathbb{C}^{*}$-action on $P / \mathbb{Z}_{m}$ which acts by translations on $C$ and which lifts to $X$. It follows that there is a canonical choice of contraction of the exceptional loci $E$ which produces $P / \mathbb{Z}_{m}$. Next we use the fact that the $\mathbb{C}^{*}$-actions on $X$ and $X^{\prime}$ are unique (up to scale) by Lemma 12. It follows that the biholomorphism between $X$ and $X^{\prime}$ must match up the $\mathbb{C}^{*}$-actions (up to scale) and so also the neighbourhoods of $E$ and $E^{\prime}$. It follows that the biholomorphism induces a biholomorphism $P / \mathbb{Z}_{m} \cong P^{\prime} / \mathbb{Z}_{m}$.

By construction, the orbifold universal cover of $P / \mathbb{Z}_{m}$ is $\operatorname{SL}(2, \mathbb{C})$ and that of $N$ is $H^{3}$ and both are obtained as quotients by the action of the same group. Hence the orbifold fundamental groups of $P / \mathbb{Z}_{m}$ f and $N$ coincide. So $P / \mathbb{Z}_{m} \cong P^{\prime} / \mathbb{Z}_{m}$ induces an isomorphism between the orbifold fundamental groups of $N$ and $N^{\prime}$ and hence, by Mostow rigidity, an isometry between $N$ and $N^{\prime}$.

\section{A symplectic example}

In this section we will explain how a similar approach-passing from a hyperbolic orbifold to a symplectic manifold via a crepant resolution-can be used to build a simply connected symplectic manifold with $c_{1}=0$ which does not admit a compatible Kähler structure.

We content ourselves here with a single example for which it is not hard to find a crepant resolution because of the simple nature of the singularities. In Section 6 we describe an infinite sequence of hyperbolic 4-orbifolds with slightly more complicated singularities to which this procedure could be applied.

\subsection{The Davis manifold}

Our construction is based on a beautiful hyperbolic 4-manifold called the Davis manifold $M$ (see Davis [11] as well as the description in Ratcliffe-Tschantz [34] which also computes the homology groups of $M$ ). The key fact for us is that $M$ admits an isometric involution which kills the fundamental group. This is analogous to the role played in the Kummer construction by the involution $z \mapsto-z$ on an abelian surface.

The manifold $M$ is built using a regular polytope called the 120-cell (or hecatonicosachoron). The 120-cell is a four-dimensional regular solid with 120 three-dimensional faces, the "cells", each of which is a solid dodecahedron. Each edge is shared by 
3 dodecahedra and each vertex by 4 dodecahedra. In total, the 120-cell has 600 vertices, 1200 edges and 720 pentagonal faces. Take a hyperbolic copy $P \subset H^{4}$ of the $120-$ cell in which the dihedral angles are $2 \pi / 5$. For each pair of opposite dodecahedral faces of $P$ there is a unique hyperbolic reflection which identifies them. Gluing opposite faces via these reflections gives the hyperbolic four-manifold $M$.

All vertices of the polytope are identified to a single point of $M$, which inherits a cell decomposition with one 0 -cell, sixty 1-cells, one-hundred and forty-four 2-cells sixty 3-cells and one 4-cells. (See Ratcliffe-Tschantz [34] for more details on the cell decomposition.)

The central involution of $H^{4}$ which fixes the centre of $P$ preserves both $P$ and the identifications of opposite faces, hence it gives an isometric involution $\sigma$ of $M$. Our symplectic construction will begin with the resulting orbifold $M / \sigma$, which we call the Davis orbifold.

To analyse the fixed points of $\sigma$ it is helpful to use the so-called "inside-out" isometry of $M$ (defined in [34]). To describe this, note that $P$ can be divided up into 14400 hyperbolic Coxeter simplices. The vertices of a simplex are given by taking first the centre of $P$, then the centre of one of its 1203 -faces $F$, then the centre of one of the 122 -faces $f$ of $F$, then the centre of one of the 5 edges $e$ of $f$ and, finally, one of the two vertices of $e$. Denote by $v_{1}, v_{2}, v_{3}, v_{4}, v_{5}$ one such choice. The corresponding simplex has a isometry that exchanges $v_{1}$ (the centre of $P$ ) with $v_{5}$ (a vertex of $e$ ), $v_{2}$ (the centre of $F$ ) with $v_{4}$ (the centre of an edge of $F^{\prime}$ ) and fixes $v_{3}$ (the centre of $f$ ). This isometry of the simplex extends to define the inside-out isometry of $M$, which commutes with $\sigma$.

Lemma 26 The fixed set of $\sigma$ consists of 122 points. The quotient $M / \sigma$ is simply connected as a topological space.

Proof In the interior of the 120-cell there is only one fixed point, the centre, all other fixed points of $\sigma$ lie on the image in $M$ of the boundary of $P$. Let $F$ denote the image in $M$ of a three-dimensional face of $P ; \sigma$ preserves $F$ and induces on it the symmetry of the dodecahedron given by inversion $x \mapsto-x$ with respect to its centre. So, once again, in the interior of $F$ there is only one fixed point, its centre. Considering all opposite pairs of three-dimensional faces of $M$ this gives 60 more fixed points of $\sigma$. All remaining fixed points are contained in the image in $M$ of the union of the 2-faces of $P$.

The symmetry $\sigma$ takes 2 -faces to 2 -faces. We claim next that $\sigma$ does not fix an interior point of any pentagonal 2-face. Assume for a moment that it does fix such 
a point. Then it would give an involution of the pentagon which would hence fix a vertex and so also the line joining the vertex to the centre of the polygon. The Davis manifold has two distinguished points, the centre and the image of all the vertices of the 120-cell. The assumption that $\sigma$ fixes an interior point of a pentagonal $2-$ face gives a $\sigma$-fixed tangent direction at the vertex point in $M$. However, the inside-out isometry exchanges the centre and vertex of $M$. Since $\sigma$ acts as $x \mapsto-x$ at the centre it does so also at the vertex and hence acts freely on the tangent space there. It follows that $\sigma$ does not fix an interior point of any 2 -face.

The remaining fixed points are contained in the image in $M$ of the union of the edges of $P$. Under the inside-out involution of $M$, the middles of all edges are exchanged with centres of all 3 -faces whilst the centre of $P$ is exchanged with the image in $M$ of the vertices of $P$. Since the inside-out isometry commutes with $\sigma$, this give an additional 61 fixed points of $\sigma$ making 122 in total.

We now turn to the (topological, not orbifold) fundamental group $\pi_{1}(M / \sigma)$. The map $\pi_{1}(M) \rightarrow \pi_{1}(M / \sigma)$ is surjective so we need to show its image is trivial. Consider the 60 closed geodesics $\gamma_{i}$ in $M$ going through the centre of $P$ and joining the centres of opposite faces. The deck transformations corresponding to these geodesics generate the whole of $\pi_{1}(M)$. Indeed, these deck transformations take the fundamental domain $P$ to all its 120 neighbours. Now the result follows from the fact that every loop $\sigma\left(\gamma_{i}\right)$ is contractible. Indeed, $\sigma$ restricts to an involution on each $\gamma_{i}$, so $\sigma\left(\gamma_{i}\right)$ is a segment in $M / \sigma$ which joins two fixed points of $\sigma$, hence is contractible.

\subsection{The model singularity and resolution}

Locally, the singularities of $M / \sigma$ are modelled on the quotient of $H^{4}$ by $x \mapsto-x$. (Here $x$ is the coordinate provided by the Poincaré ball model of $H^{4}$.) By Proposition 4 we know that the corresponding symplectomorphism of $R$ is given by $z \mapsto-z$ in the vector bundle fibres of $\mathcal{O}(-1) \oplus \mathcal{O}(-1)$. The quotient $(\mathcal{O}(-1) \oplus \mathcal{O}(-1)) / \mathbb{Z}_{2}$ is a Kähler Calabi-Yau orbifold with singular locus $\mathbb{C} \mathbb{P}^{1}$ corresponding to the zero section. We next describe a crepant resolution of this singularity.

\section{Lemma 27 There is a crepant resolution}

$$
\mathcal{O}(-2,-2) \rightarrow(\mathcal{O}(-1) \oplus \mathcal{O}(-1)) / \mathbb{Z}_{2}
$$

where $\mathcal{O}(-2,-2) \rightarrow \mathbb{C P}^{1} \times \mathbb{C P}^{1}$ is the tensor product of the two line bundles given by pulling back $\mathcal{O}(-2) \rightarrow \mathbb{C P}^{1}$ from either factor.

Proof Blow up the zero section of $\mathcal{O}(-1) \oplus \mathcal{O}(-1)$ to obtain the total space of $\mathcal{O}(-1,-1) \rightarrow \mathbb{C P}^{1} \times \mathbb{C P}^{1}$. The $\mathbb{Z}_{2}$-action lifts to this line bundle where it again has 
fixed locus the zero section and acts by $z \mapsto-z$ in the fibres. For such an involution on any line bundle $L$, the square gives a resolution $L^{2} \rightarrow L / \mathbb{Z}_{2}$. Hence

$$
\mathcal{O}(-2,-2) \rightarrow \mathcal{O}(-1,-1) / \mathbb{Z}_{2} \rightarrow(O(-1) \oplus \mathcal{O}(-1)) / \mathbb{Z}_{2}
$$

gives the claimed resolution

It must be emphasised that this is resolution is holomorphic. The total space of $\mathcal{O}(-2,-2)$ is a Kähler manifold with trivial canonical bundle and, away from the exceptional divisor, the map in Lemma 27 is a biholomorphism when we consider $(\mathcal{O}(-1) \oplus \mathcal{O}(-1)) / \mathbb{Z}_{2}$ with its holomorphic complex structure. However, when constructing symplectic six-manifolds from hyperbolic four-manifolds, the relevant almost complex structure and volume form on $\mathcal{O}(-1) \oplus \mathcal{O}(-1)$ (and its $\mathbb{Z}_{2}$-quotient) are not the holomorphic ones; rather we use the $\mathrm{SO}(4,1)$-invariant almost complex structure and complex volume form from Proposition 4. Lemma 27 can only be used to provide crepant resolutions of Calabi-Yau singularities modelled on the holomorphic geometry of $(\mathcal{O}(-1) \oplus \mathcal{O}(-1)) / \mathbb{Z}_{2}$ and not the $\mathrm{SO}(4,1)$-invariant almost complex structure and complex volume form of Proposition 4.

So, in order to apply Lemma 27 to resolve singularities in hyperbolic twistor spaces, we need to interpolate between the holomorphic structures near the zero section in $\mathcal{O}(-1) \oplus \mathcal{O}(-1)$ to the $\mathrm{SO}(4,1)$-invariant structures outside a small neighbourhood of the zero section. This interpolation is provided by the following result.

Lemma 28 Let $R_{\delta}$ denote the part of $R$ lying over a geodesic ball in $H^{4}$ of radius $\delta$. For any $\delta>0$, there is an $\mathrm{SO}(4)$-invariant compatible almost complex structure $J$ on $R$ and an $\mathrm{SO}(4)$-invariant nowhere-vanishing section $\Omega$ of the $J$-canonical bundle such that:

- Over $R_{\delta}, J$ and $\Omega$ agree with the standard holomorphic structures.

- Over $R \backslash R_{2 \delta}, J$ and $\Omega$ agree with the $\mathrm{SO}(4,1)$-invariant structures from Proposition 4.

Proof As is standard, an SO(4)-invariant interpolation between the "inside" and "outside" Hermitian metrics gives the existence of $J$.

To produce $\Omega$ we start with a description of the $\mathrm{SO}(4)$-action away from the zerosection $R_{0}$. The stabiliser of a point $p \in R \backslash R_{0}$ is a circle $S_{p}^{1} \subset \mathrm{SO}(4)$ and the orbit of $p$ is 5-dimensional (in fact, isomorphic as an $\mathrm{SO}(4)$-space to the unit tangent bundle of $S^{3}$ ). The lift of a geodesic ray out of the origin in $H^{4}$ meets each $\mathrm{SO}(4)-$ orbit in a unique point, giving a section for the action. We interpolate between the 
holomorphic and hyperbolic complex volume forms along the relevant portion of this lifted ray and then use the $\mathrm{SO}(4)$-action to extend the resulting 3 -form to the whole of $R$. In order for this to work it is sufficient that at every point $p \in R \backslash R_{0}$ the action of $S_{p}^{1}$ on the fibre of the $J$-canonical bundle at $p$ is trivial. But since the weight is integer valued and continuous it is constant on $R \backslash R_{0}$ so we can compute it for some $p$ outside of $R_{2 r}$ where everything agrees with the hyperbolic picture. Here we already have an $\mathrm{SO}(4)$-invariant (hence $S_{p}^{1}$-invariant) complex volume-form so the weight is zero as required.

\subsection{The twistor space of the Davis orbifold}

With Lemma 27 and Lemma 28 in hand, we can now take a crepant resolution of the twistor space of the Davis orbifold $M / \sigma$. Let $Z \rightarrow M$ denote the twistor space of the Davis manifold The involution $\sigma$ lifts to an involution of $Z$ which we still denote $\sigma . Z / \sigma$ is a symplectic orbifold with singularities along 122 copies of $\mathbb{C P}^{1}$, each modelled on $\mathcal{O}(-1) \oplus \mathcal{O}(-1) / \mathbb{Z}_{2}$.

Let $\delta$ be a positive number small enough that the geodesic balls in $M$ of radius $2 \delta$ centred on the $\sigma$-fixed points are embedded and disjoint. Then, by Lemma 28 , on $Z$ we can find a new almost complex structure $J$ and complex volume form $\Omega$ such that outside the geodesic $2 \delta$-balls they agree with the hyperbolic structures coming from Proposition 4 , whilst inside the balls of radius $\delta$ they agree with the holomorphic structures coming from the holomorphic geometry of $\mathcal{O}(-1) \oplus \mathcal{O}(-1)$. It follows from the SO(4)-invariance in Lemma 28 that $J$ and $\Omega$ are $\sigma$-invariant.

In this way the quotient $Z / \sigma$ is a symplectic orbifold with an almost complex structure and complex volume form which are modelled near the singular curves on the holomorphic geometry of $\mathcal{O}(-1) \oplus \mathcal{O}(-1) / \mathbb{Z}_{2}$. It follows from Lemma 27 that there is a resolution $\hat{Z} \rightarrow Z / \sigma$ in which the singular curves have been replaced by copies of $\mathbb{C P}^{1} \times \mathbb{C P}^{1}$ with normal bundle $\mathcal{O}(-2,-2)$; moreover, $\hat{Z}$ carries an almost complex structure $\widehat{J}$ and complex volume form $\widehat{\Omega}$ so that $c_{1}(\hat{Z}, \widehat{J})=0$.

Finally we need to define the symplectic structure on $\hat{Z}$. Pulling back the symplectic form via $\hat{Z} \rightarrow Z$ gives a symplectic form on the complement of the exceptional divisors. To extend it we use a standard fact about resolutions in Kähler geometry. Given any neighbourhood $U$ of the zero locus in $\mathcal{O}(-2,-2)$, there is a Kähler metric on $\mathcal{O}(-2,-2)$ for which the projection to $\mathcal{O}(-1) \oplus \mathcal{O}(-1) / \mathbb{Z}_{2}$ is an isometry on the complement of $U$. (This amounts to the fact that the zero locus has negative normal bundle.)

So, in the model, the pullback of the symplectic form extends over the exceptional divisor in a way compatible with holomorphic complex structure. Taking $U$ sufficiently 
small and doing this near all 122 exceptional divisors defines a symplectic form $\omega$ on $\hat{Z}$ which is compatible with $\hat{J}$.

\subsection{Simple connectivity and non-Kählerity}

This section proves that $\hat{Z}$ is simply connected and admits no compatible integrable complex structure. The second fact will follow from the first and the fact that $b_{3}(\widehat{Z})=0$.

\section{Lemma $29 \hat{Z}$ is simply connected.}

Proof We first apply Theorem 13 (with $n=2$ ) to the map $Z / \sigma \rightarrow M / \sigma$. The fibres are $2-$ spheres and we see that $\pi_{1}(Z / \sigma)=1$. Next we apply Theorem 13 to $\hat{Z} \rightarrow Z / \sigma$. This time the fibres are points or 2 -spheres and we deduce that $\pi_{1}(\hat{Z})=1$.

To prove that $b_{3}(\hat{Z})=0$ we invoke a lemma of McDuff on the cohomology of manifolds obtained by symplectic blow-ups.

Lemma 30 (McDuff [29]) Let $X$ be a symplectic manifold and $C \subset X$ a smooth symplectic submanifold of codimension $2 k$. Let $\tilde{X}$ denote the blow-up of $X$ along $C$. Then the real cohomology of $\tilde{X}$ fits into a short exact sequence of graded vector spaces

$$
0 \rightarrow H^{*}(X) \rightarrow H^{*}(\tilde{X}) \rightarrow A^{*} \rightarrow 0
$$

where the first arrow is pullback via $\tilde{X} \rightarrow X$ and where $A^{*}$ is a free module over $H^{*}(C)$ with one generator in each dimension $2 j, 1 \leq j \leq k-1$.

\section{Lemma $31 b_{3}(\hat{Z})=0$.}

Proof Recall that $Z \rightarrow M$ is the twistor space of the Davis manifold. We first blow up the 122 fibres which lie over the fixed points of $\sigma$ to obtain the new manifold $\tilde{Z}$. It follows from Lemma 30 that pulling back cohomology via $\tilde{Z} \rightarrow Z$ induces an isomorphism $H^{3}(\widetilde{Z}) \cong H^{3}(Z)$.

Next, notice that $\sigma$ lifts to $\tilde{Z}$ and that $\hat{Z}=\tilde{Z} / \sigma$. We now show that $\sigma$ acts as -1 on $H^{3}(\tilde{Z})$. To see this, consider the action of $\sigma$ on the Davis manifold $M$. It acts on $H^{1}(M)$ as -1 and hence also as -1 on $H^{3}(M)$. Now $Z \rightarrow M$ is a sphere bundle so, by Leray-Hirsch, $H^{*}(Z)$ is a free module over $H^{*}(M)$ with a single generator in degree 2 corresponding to the first Chern class of the vertical tangent bundle. This generator is preserved by $\sigma$, so $\sigma$ acts as -1 on $H^{3}(Z)$ and hence also as -1 on $H^{3}(\widetilde{Z})$. From this we deduce that $H^{3}(\hat{Z})=0$. For if it contained a nonzero element, the pullback to $\widetilde{Z}$ would be a $\sigma$-invariant element of $H^{3}(\widetilde{Z})$. 
Corollary 32 There is no Kähler structure on $\hat{Z}$ with $c_{1}=0$. In particular, the symplectic structure on $\hat{Z}$ described above admits no compatible complex structure.

Proof For a Kähler manifold, the vanishing of $b_{1}$ implies the Picard torus is trivial. Now $c_{1}=0$ implies the existence of a holomorphic volume form, hence $b_{3} \geq 2$. (For more details see Lemma 2.13 of [15].)

Note that whilst we have shown that there is no Calabi-Yau Kähler structure on $\hat{Z}$, it is not clear, to us at least, whether or not $\hat{Z}$ admits a Kähler structure when one does not place a restriction on $c_{1}$.

\section{Some symplectic "Fano" manifolds}

In this section we explain how hyperbolic geometry in higher dimensions leads to symplectic manifolds for which the first Chern class is a positive multiple of the symplectic class, non-Kähler analogues of Fano manifolds.

\subsection{Hyperbolic geometry in even dimensions}

The passage from hyperbolic 4-manifolds to symplectic 6-manifolds can be generalised to every even dimension, with hyperbolic $2 n$-manifolds giving symplectic $n(n+1)$-manifolds. This was first explained via twistors, by Reznikov [36] (although Reznikov did not consider the first Chern class of his examples). Here we give an alternative description in terms of coadjoint orbits, as in Section 2.3.3.

We consider a certain coadjoint orbit of $\operatorname{SO}(2 n, 1)$ (for each $n=1,2, \ldots$ ). The Lie algebra $\mathfrak{s o}(2 n, 1)$ consists of $(2 n+1) \times(2 n+1)$ matrices of the form

$$
\left(\begin{array}{ll}
0 & u^{t} \\
u & A
\end{array}\right)
$$

where $u$ is a column vector in $\mathbb{R}^{2 n}$ and $A \in \mathfrak{s o}(2 n)$. Those elements with $u=0$ generate $\mathrm{SO}(2 n) \subset \mathrm{SO}(2 n, 1)$. We consider the following normalisation of the Killing form on $\mathfrak{s o}(2 n, 1)$ :

$$
(X, Y) \mapsto-\frac{1}{2} \operatorname{Tr}(X Y) .
$$

This is nondegenerate and so gives an equivariant isomorphism $\mathfrak{s o}(2 n, 1) \cong \mathfrak{s o}(2 n, 1)^{*}$. As in Section 2.3.3, we consider the orbit of

$$
\xi=\left(\begin{array}{ll}
0 & 0 \\
0 & J_{0}
\end{array}\right)
$$


where $J_{0} \in \mathfrak{s o}(2 n)$ is a choice of almost complex structure on $\mathbb{R}^{2 n}$ (ie, $J_{0}^{2}=-1$ ). The stabiliser of $\xi$ is a copy of $\mathrm{U}(n)$ inside $\mathrm{SO}(2 n)$. We write $Z_{2 n}$ for the coadjoint orbit of $\xi$.

So the theory of coadjoint orbits gives us a symplectic manifold of dimension $n(n+1)$, which we write $Z_{2 n} \cong \mathrm{U}(n) \backslash \mathrm{SO}(2 n, 1)$. It fibres over hyperbolic space $H^{2 n} \cong$ $\mathrm{SO}(2 n) \backslash \mathrm{SO}(2 n, 1)$ with fibre isomorphic to the space $\mathrm{U}(n) \backslash \mathrm{SO}(2 n)$ of orthogonal complex structures on $\mathbb{R}^{2 n}$ inducing a fixed orientation. In other words, it is the twistor space of $H^{2 n}$.

We are interested in the first Chern class of $Z_{2 n}$. Our goal is to show that $c_{1}\left(Z_{2 n}\right)=$ $(n-2)[\omega]$. We will do this in two steps. Firstly, we will investigate the class $[\omega]$. We will prove:

\section{Lemma 33}

- There is a Hermitian line bundle $L \rightarrow Z_{2 n}$ and a lift of the $\mathrm{SO}(2 n, 1)$-action on $Z_{2 n}$ to an action on $L$ by bundle isometries.

- There is a unitary connection in $L$ whose curvature defines the symplectic form $\omega$ on $Z_{2 n}$. Hence $[\omega]=c_{1}(L)$. Moreover, this connection, and hence $\omega$, is invariant under the action of $\mathrm{SO}(2 n, 1)$.

Next we will consider the first Chern class of $Z_{2 n}$. We will prove:

\section{Lemma 34}

- There is an $\mathrm{SO}(2 n, 1)$-invariant almost complex structure on $Z_{2 n}$ which is compatible with $\omega$. Note that the invariance implies that the corresponding canonical bundle $K$ is acted on by $\mathrm{SO}(2 n, 1)$, lifting the action on $Z_{2 n}$.

- There is an $\mathrm{SO}(2 n, 1)$-equivariant isomorphism $K \cong L^{2-n}$.

It is also possible to describe $L$ in terms of the fibration $Z_{2 n} \rightarrow H^{2 n}$; see Remark 37 for these alternative descriptions. From Lemma 33 and Lemma 34 it follows that $c_{1}\left(Z_{2 n}\right)=(n-2) c_{1}(L)=(n-2)[\omega]$.

We focus now on Lemma 33. This result is a special case of the theory of integral coadjoint orbits. We recall briefly the general theory here. Details and proofs can be found in, for example, Wallach [43].

Let $G$ be a Lie group, $\xi \in \mathfrak{g}^{*}$ with stabiliser $H$ and let $\mathfrak{h}$ be the Lie algebra of $H$. The restriction of $\xi$ to $\mathfrak{h}$ defines a map $\xi: \mathfrak{h} \rightarrow \mathbb{R}$ which is a Lie algebra homomorphism, by virtue of the fact that $H$ fixes $\xi$. Regarding $\mathbb{R}$ as the Lie algebra of $U(1)$, one can ask if this Lie algebra homomorphism is induced by a group homomorphism $H \rightarrow U(1)$. 
Definition 35 The coadjoint orbit of $\xi$ is called integral if the Lie algebra homomorphism $\xi: \mathfrak{h} \rightarrow \mathbb{R}$ is the derivative of a group homomorphism $\rho: H \rightarrow U(1)$.

When the orbit $\mathcal{O}(\xi)$ is integral, the general theory gives us exactly what we want. Firstly there is a Hermitian line bundle $L \rightarrow \mathcal{O}(\xi)$ and a lift of the $G$-action on $\mathcal{O}(\xi)$ to $L$ by bundle isometries. Secondly, there is a $G$-equivariant connection in $L$ whose curvature is $\omega$.

We outline how $L$ and the connection arise, referring eg, to [43] for the details. We first describe the construction of $L$. The orbit $\mathcal{O}(\xi) \cong H \backslash G$ is the base of a principal $H$ bundle $G \rightarrow H \backslash G$. Given the representation $\rho: H \rightarrow U(1)$, we define the Hermitian line bundle $L \rightarrow \mathcal{O}(\xi)$ by $L=G \times{ }_{\rho} \mathbb{C}$, where we have taken the quotient of $G \times \mathbb{C}$ by the diagonal action of $H$ given by $h \cdot(g, v)=\left(h g, \rho(h)^{-1} v\right)$. Notice that the action of $G$ by right multiplication on $G \times \mathbb{C}$ (with $G$ acting trivially on $\mathbb{C}$ ) commutes with the $H$ action and so descends to give a $G$ action on $L$ covering the action on $H \backslash G$.

We next describe the connection. At each point $p \in L$, the infinitesimal $G$-action gives a map $r_{p}: \mathfrak{g} \rightarrow T_{p} L$. We restrict $r_{p}$ to the kernel of the map $\xi: \mathfrak{g} \rightarrow \mathbb{R}$. This gives a subspace $r_{p}(\operatorname{ker} \xi)$ in $T_{p} L$ which one checks is transverse to the fibres of $L \rightarrow K \backslash G$ and defines a unitary connection with curvature $\omega$. By construction it is $G$-invariant.

We are now have the background to prove Lemma 33.

Proof of Lemma 33 From the above discussion, it suffices to prove that $Z_{2 n}$ is an integral coadjoint orbit. That is, we must prove that the map $\xi: \mathfrak{h} \rightarrow \mathbb{R}$ integrates to a group homomorphism $H \rightarrow U(1)$. Elements of $\mathfrak{h}$ have the form

$$
\left(\begin{array}{ll}
0 & 0 \\
0 & A
\end{array}\right)
$$

where $A \in \mathfrak{s o}(2 n)$ commutes with $J_{0}$. (The definition of $\xi$ requires a choice of almost complex structure $J_{0}$ on $\mathbb{R}^{2 n}$.) Recall that we used the Killing form $(X, Y) \mapsto$ $-\frac{1}{2} \operatorname{Tr}(X Y)$ to identify $\mathfrak{s o}(2 n, 1) \cong \mathfrak{s o}(2 n, 1)^{*}$. So the map $\xi: \mathfrak{h} \rightarrow \mathbb{R}$ is given by

$$
A \mapsto-\frac{1}{2} \operatorname{Tr}\left(A J_{0}\right) .
$$

On the other hand, using $J_{0}$ to identify $\mathbb{R}^{2 n} \cong \mathbb{C}^{n}$, those elements $A$ of $\mathfrak{s o}(2 n)$ which commute with $J_{0}$ become identified with skew-Hermitian matrices $\mathfrak{u}(n)$. If we write $A_{\mathbb{C}}$ for $A$ thought of as a skew-Hermitian matrix, then

$$
\operatorname{Tr}\left(A J_{0}\right)=2 i \operatorname{Re} \operatorname{Tr}\left(A_{\mathbb{C}}\right) .
$$

From here we see that the map $\xi: \mathfrak{h} \rightarrow \mathbb{R}$ is identified with the map $-\operatorname{Tr}: \mathfrak{u}(n) \rightarrow i \mathbb{R}$. This integrates to the homomorphism $\mathrm{U}(n) \rightarrow U(1)$ given by $M \mapsto(\operatorname{det} M)^{-1}$ and so the coadjoint orbit is integral as required. 
We now turn to the first Chern class of $Z_{2 n}$ and the proof of Lemma 34. For this we first need to pick a compatible almost complex structure. The same proof as in Lemma 5 gives the following result.

Lemma 36 There is an isomorphism of $U(n)$-representation spaces:

$$
\mathfrak{s o}(2 n, 1) \cong \mathfrak{u}(n) \oplus \Lambda^{2}\left(\mathbb{C}^{n}\right)^{*} \oplus \mathbb{C}^{n} .
$$

Given a point $z \in Z_{2 n}$ with stabiliser $\mathrm{U}(n) \subset \mathrm{SO}(2 n, 1)$ there is a $U(n)$-equivariant isomorphism

$$
T_{z} \cong \Lambda^{2}\left(\mathbb{C}^{n}\right)^{*} \oplus \mathbb{C}^{n},
$$

in which the $\Lambda^{2}\left(\mathbb{C}^{n}\right)^{*}$ summand is tangent to the fibre of the projection $Z_{2 n} \rightarrow H^{2 n}$.

We are now in a position to give the proof of Lemma 34

Proof of Lemma 34 As in Lemma 6, by $\mathrm{U}(n)$-equivariance, the symplectic form on $T_{z}$ is proportional under (5) to the form induced by the Euclidean structure on $\mathbb{C}^{n}$. To show this constant of proportionality is positive, first we check that the forms are genuinely equal in the case $n=1$, where $\mathfrak{s o}(2,1) \cong \mathfrak{u}(1) \oplus \mathbb{C}$ as a $\mathrm{U}(1)$-representation. This amounts to the fact that $Z_{2}=H^{2}$ with symplectic form the hyperbolic area form. Next, we use induction and the fact that the decompositions of $\mathfrak{s o}(2 n, 1)$ and $\mathfrak{s o}(2 n+2,1)$ from Lemma 36 are compatible with the obvious inclusions of the summands induced by a choice of $\mathbb{C}^{n} \subset \mathbb{C}^{n+1}$.

Having seen that the isomorphism of (5) is symplectic, we define a compatible $\mathrm{SO}(2 n, 1)$-invariant almost complex structure on $Z_{2 n}$ by declaring (5) to be a complex linear isomorphism. This gives the first part of Lemma 34.

For the second part, we recall that $Z_{2 n} \cong \mathrm{U}(n) \backslash \mathrm{SO}(2 n, 1)$ is the base of a principal $\mathrm{U}(n)$-bundle $Q \rightarrow Z_{2 n}$. What Lemma 36 tells us is that the tangent bundle of $Z_{2 n}$ is isomorphic to the complex vector bundle associated to $Q$ via the natural action of $\mathrm{U}(n)$ on $\Lambda^{2}\left(\mathbb{C}^{n}\right)^{*} \oplus \mathbb{C}^{n}$. If follows that the anticanonical bundle of $Z_{2 n}$ is the complex line bundle associated to $Q$ via the determinant of this representation.

The weight of $\mathrm{U}(n)$ acting on $\Lambda^{2}\left(\mathbb{C}^{n}\right)$ is $n-1$. This can be checked by induction, via the isomorphism of representations $\Lambda^{2}\left(\mathbb{C}^{n+1}\right) \cong \Lambda^{2}\left(\mathbb{C}^{n}\right) \oplus \mathbb{C}^{n}$. Hence the weight of $\mathrm{U}(n)$ acting on $\Lambda^{2}\left(\mathbb{C}^{n}\right)^{*} \oplus \mathbb{C}^{n}$ is $2-n$. It follows that the anticanonical bundle is associated to $Q$ via the representation $\mathrm{U}(n) \rightarrow S^{1}$ given by $M \mapsto(\operatorname{det} M)^{2-n}$.

Meanwhile, from the general theory of integral coadjoint orbits and the proof of Lemma 33 , the Hermitian line bundle is built from $Q$ via the representation $\mathrm{U}(n) \rightarrow U(1)$ given 
by $M \mapsto(\operatorname{det} M)^{-1}$. It follows that $K \cong L^{2-n}$. Moreover, the description of both $K$ and $L^{2-n}$ as bundles associated to $Q$ via the same representation $\mathrm{U}(n) \rightarrow U(1)$ means that this isomorphism can be chosen to be $\mathrm{SO}(2 n, 1)$-equivariant.

Remark 37 In fact, this proof gives two additional alternative descriptions of $L$. From Lemma 36 we see that the tangent bundle to $Z_{2 n}$ splits $T Z_{2 n}=\operatorname{Ver} \oplus$ Hor as a sum of complex bundles. Ver is tangent to the fibres of $Z_{2 n} \rightarrow H^{2 n}$ whilst Hor is transverse. Considering weights as in the proof of Lemma 34 shows that $L$ is isomorphic to $\Lambda^{n}$ Hor $^{*}$ or that $L^{n-1}$ is isomorphic to $\Lambda^{n(n-1) / 2} \operatorname{Ver}($ where $n(n-1) / 2$ is the rank of Ver).

Note that the fibres of $Z_{2 n} \rightarrow H^{2 n}$ are all homogeneous symplectic manifolds of the form $\mathrm{U}(n) \backslash \mathrm{SO}(2 n)$. In fact, this space is well known to be an algebraic Fano manifold, polarised by the top exterior power of its tangent bundle. The above weight calculation shows that the restriction of $L$ to each fibre is a positive power of this Fano polarisation.

Finally, we remark that this vertical-horizontal splitting can be seen from the twistorial point of view. The fibre of $Z_{2 n}$ over $x$ is the space of all orthogonal linear complex structures on $T_{x} H^{2 n}$ compatible with a fixed choice of orientation. The Levi-Civita connection of the hyperbolic metric determines the horizontal distribution Hor. Moreover, at a point $p \in Z_{2 n}$, the horizontal space Hor Ho $_{p}$ is simply the tangent space downstairs, $T_{x} H^{2 n}$, equipped with the complex structure corresponding to $p$.

\subsection{Compact quotients}

The $\operatorname{SO}(2 n, 1)$-equivariance in Lemmas 33 and 34 imply the following result.

Lemma 38 Let $\Gamma$ be a torsion-free lattice in $\operatorname{SO}(2 n, 1)$ and write $X=Z_{2 n} / \Gamma$ for the quotient. (Note the lack of torsion in $\Gamma$ implies $X$ is smooth).

- Both the Hermitian line bundle $L \rightarrow Z_{2 n}$ and the unitary connection in $L$ descend to give a line bundle $E \rightarrow X$ and a unitary connection in $E$ whose curvature is a symplectic form $\omega$ representing $c_{1}(E)$.

- The compatible almost complex structure on $Z_{2 n}$ descends to $X$ to give a compatible almost complex structure whose associated canonical bundle $K$ satisfies $K \cong E^{2-n}$. In particular, $c_{1}(X)=(n-2)[\omega]$.

This means we can find compact examples of symplectic manifolds for which $c_{1}(X)$ is a positive multiple of $[\omega]$. Let $\Gamma \subset \mathrm{SO}(2 n, 1)$ be the fundamental group of a compact hyperbolic $2 n$-manifold $M$. $\Gamma$ acts by symplectomorphisms on $Z_{2 n}$ to give as 
quotient a symplectic manifold of dimension $n(n+1)$. It fibres $Z_{2 n} / \Gamma \rightarrow H^{2 n} / \Gamma$ over $M$ as the twistor space of $M$. When $n \geq 3$, these are compact symplectic manifolds for which $c_{1}$ is a positive multiple of $[\omega]$. Such manifolds can never be Kähler since they have hyperbolic fundamental group (see Section 2 of [2]). Indeed, Kähler Fano manifolds are even simply connected (see page 126 of [24]). These examples (originally appearing in Reznikov's article [36]) are, to the best of our knowledge, the first non-Kähler symplectic "Fano" manifolds. The lowest dimension which can be achieved in this way is 12 . In this case, the fibration over $M^{6}$ has fibres $\mathbb{C P}^{3} \cong \mathrm{SO}(6) / \mathrm{U}(3)$.

\section{Concluding remarks}

The constructions presented here seem, to us at least, to lead several natural questions. We describe some of these below.

\subsection{Simply connected symplectic examples}

Whilst we only give one example of a simply connected symplectic manifold with $c_{1}=0$, we plan to exploit a similar construction to produce simply connected $6-$ dimensional examples with arbitrarily large Betti numbers (see the forthcoming article [14]). By comparison, note that it is still unknown if there are infinitely many topologically distinct Kähler Calabi-Yau manifolds of fixed dimension.

First we describe an infinite sequence of simply connected compact symplectic orbifolds with $c_{1}=0$. They come from hyperbolic orbifold metrics on $S^{4}$, built using Coxeter polytopes. Recall that a hyperbolic Coxeter polytope is a convex polytope with totally geodesic boundary in $H^{n}$ and whose dihedral angles are $\pi / k$ for $k \in \mathbb{N}$; the polytope is said to be right-angled if all dihedral angles are $\pi / 2$.

Lemma 39 Let $P$ be an $n$-dimensional compact hyperbolic Coxeter polytope. Doubling $P$ gives a hyperbolic orbifold metric on $S^{n}$.

Proof Let $G$ be the group of isometries of $H^{n}$ generated by reflections in the faces of $P$ and let $G^{\prime}$ be the subgroup of $G$ of index two consisting of all orientation preserving elements. Then $H^{n} / G^{\prime}$ is the double of $P$. Indeed the fundamental domain of $G$ is $P$ itself, whilst the fundamental domain of $G^{\prime}$ is $P \cup P^{\prime}$; where $P^{\prime}$ is the reflection of $P$ in a face. Identifying further the faces of $P \cup P^{\prime}$ via $G^{\prime}$ we obtain the double of $P$. The double is homeomorphic to $S^{n}$, since $P$ is homeomorphic to a closed $n$-ball. 
Up to dimension 6, compact hyperbolic Coxeter polytopes are known to be abundant.

Theorem 40 (Potyagilo-Vinberg [33]; Allcock [1]) In all dimensions up to 6 there exist infinitely many compact hyperbolic Coxeter polytopes. Moreover, in dimensions up to 4 there are infinitely many compact hyperbolic right-angled polytopes.

We are interested in the case of 4 and 6 dimensions. The right angled 4-dimensional polytopes are constructed by Potyagilo-Vinberg [33]. Here one uses that there is a hyperbolic 120 -cell with dihedral angles $\pi / 2$. Gluing it to itself along a 3 -face (via the reflection in that face) produces a new right-angled polytope. This procedure can be repeated giving infinitely many examples. An infinite family of 6-dimensional polytopes is constructed by Allcock [1].

Doubling the 4-dimensional hyperbolic Coxeter polytopes gives infinitely many simply connected symplectic 6-orbifolds with $c_{1}=0$.

When the polytope is doubled, the hyperbolic metric extends smoothly across the 3 -faces; the singularities correspond to the 2-skeleton of the polytope. To understand the singularities in the twistor space, consider the positive "octant" $x_{1}, x_{2}, x_{3}, x_{4} \geq 0$ in $\mathbb{R}^{4}$, ie, the infinitesimal model for the vertex of a right-angled Coxeter polytope. The $\left(x_{1}, x_{2}\right)$-plane lifts to two planes $L, L^{\prime}$ in the twistor space, corresponding to the two compatible complex structures on $\mathbb{R}^{4}$ for which the $\left(x_{1}, x_{2}\right)$-plane is a complex line. Reflection in the $\left(x_{1}, x_{2}\right)$-plane lifts to the twistor space where it fixes $L, L^{\prime}$ pointwise. The other coordinate 2 -planes behave similarly. The lifts of coordinate 2 -planes are some of the points with nontrivial stabiliser under the action on the twistor space generated by the reflections. The only other points with nontrivial stabiliser are those on the twistor line over the origin. This line is fixed pointwise by the composition of reflection in one 2-plane with reflection in the orthogonal 2-plane.

It follows that the orbifold singularities of the twistor space of a doubled right-angled Coxeter polytope are of two sorts. The generic orbifold point has structure group $\mathbb{Z}_{2}$ and is modelled on the quotient of $\mathbb{C}^{3}$ by $\left(z_{1}, z_{2}, z_{3}\right) \mapsto\left(z_{1},-z_{2},-z_{3}\right)$. These correspond either to the lifted coordinate 2 -planes or the central twistor line in the above picture. Then there are isolated points in the singular locus, where three surfaces of generic orbifold points meet. Here the structure group is $\left(\mathbb{Z}_{2}\right)^{2}$. This is most symmetrically described via the action of $\left(\mathbb{Z}_{2}\right)^{3}$ on $\mathbb{C}^{3}$ where each generator changes the sign on one of the three coordinate 2 -planes in $\mathbb{C}^{3}$; the diagonal $\mathbb{Z}_{2}$ acts trivially so the action factors through $\left(\mathbb{Z}_{2}\right)^{2}$. Such points correspond in the picture above to the intersection of the twistor line at the origin with the lifts of two orthogonal coordinate 2-planes.

The concrete description of these singularities means that it is possible to find symplectic crepant resolutions "by hand", as it was for the Davis orbifold. This gives an infinite 
collection of simply connected symplectic manifolds with $c_{1}=0$ [14]. Meanwhile, the twistor spaces of the 6-orbifolds of Allcock give an infinite collection of simply connected symplectic Fano 12-orbifolds. It is natural to ask if these admit symplectic Fano resolutions, although this looks much harder to answer than the corresponding question for the 6-orbifolds.

On the subject of symplectic resolutions, we mention the recent work of NiederkrügerPasquotto $[32 ; 31]$, which gives a systematic approach to the resolution of symplectic orbifolds arising via symplectic reduction, although they do not consider discrepancy.

\subsection{Possible diversity of symplectic 6-manifolds with $c_{1}=0$}

On the subject of hyperbolic orbifolds, there is a much more general existence question, which we learnt from Gromov:

Question 41 Is there any restriction on the manifolds which can be obtained as quotients of $H^{n}$ by a cocompact discrete subgroup of $\mathrm{O}(n, 1)$ ?

(The subgroup is allowed to have torsion, of course.) For $n=2$ and 3 all compact manifolds can be obtained as quotients ( $n=2$ is straightforward whilst $n=3$ uses geometrization). The relationship between hyperbolic and symplectic geometry outlined here gives additional motivation to try to answer the question in dimension four. For example, can any finitely presented group be the fundamental group of such a quotient? The 4-dimensional quotients give rise to 6-dimensional symplectic orbifolds with $c_{1}=0$ and in this way one might ambitiously hope to approach the problem of which groups can appear as the fundamental group of a symplectic manifold with $c_{1}=0$. Of course, even if one had the orbifolds, they would still need to be resolved. In algebraic geometry, crepant resolutions of threefolds always exist, thanks to the work of Bridgeland-King-Reid [7]. Even independently of the hyperbolic orbifold approach described here, it would be interesting to know what holds in the symplectic setting.

\subsection{Rational curves in hyperbolic twistor spaces}

We conclude our discussion of the symplectic examples with a brief look at the genus zero Gromov-Witten invariants of hyperbolic twistor spaces.

Lemma 42 Let $\mathbb{C P}^{1} \rightarrow Z_{2 n}$ be pseudoholomorphic with respect to the $\mathrm{SO}(2 n, 1)$ invariant almost complex structure defined above. Then the image lies entirely in a fibre of $Z_{2 n} \rightarrow H^{2 n}$. 
Proof A theorem of Salamon [37] (Eells-Salamon [12] in the case $n=2$ ) shows that the image in $H^{2 n}$ of a pseudoholomorphic curve in $Z_{2 n}$ is a minimal surface. Since $H^{2 n}$ contains no minimal spheres the result follows.

It follows that there are pairs of points in $Z_{2 n}$ (or any of its compact quotients) which do not lie on a pseudoholomorphic rational curve. This is in stark contrast to the situation for Kähler Fano manifolds. Of course, we consider here a particular almost complex structure. The symplectic definition of rational connectivity (see Li-Ruan [27]) involves the nonvanishing of certain genus zero Gromov-Witten invariants. A weaker property than rational connectivity is that of being uniruled. In algebraic geometry a variety is called uniruled if each point is contained in a rational curve. Again, the definition in symplectic geometry involves a statement about genus zero Gromov-Witten invariants. Li-Ruan [27] have asked if all symplectic Fano manifolds are uniruled in this sense.

We content ourselves here by mentioning that the above restriction on the image of rational curves suggests an approach to computing the genus zero Gromov-Witten invariants of the twistor spaces of hyperbolic $2 n$-manifolds. Whilst the invariant almost complex structure is not generic, the obstruction bundle should be describable in simple terms. Indeed, using this lemma it should be possible to localise calculations to the case of a curve in the fibre of $Z_{2 n} \rightarrow H^{2 n}$ and exploit the action of the $\operatorname{SO}(2 n)$ of isometries fixing the point in $H^{2 n}$.

\subsection{Miles Reid's fantasy}

In [35], Miles Reid asked a question, which is now referred to as Reid's fantasy:

Question 43 (Reid [35]) Consider the moduli space of complex structures on the manifold $\left(S^{3} \times S^{3}\right)^{\# N}$ with $K \cong \mathcal{O}$ which are deformations of Moishezon spaces. Is it true that for large $N$ this space is irreducible?

Do all simply connected Kähler Calabi-Yau threefolds appear as small resolutions of 3 -folds with double points lying on the boundary of these moduli spaces?

The complex threefolds that we obtain do not appear directly in Reid's fantasy and indeed seem to be of a very different nature. There is no visible mechanism that would enable one to connect these examples in any way to Moishezon manifolds. It seems more reasonable that the structures we construct on $2\left(S^{3} \times S^{3}\right) \#\left(S^{2} \times S^{4}\right)$ belong to an infinite family of disconnected components of complex structures with $K \cong \mathcal{O}$. If this were true, it would show that Reid was wise to limit himself in his fantasies when he wrote "I aim to consider only analytic threefolds which are deformations of Moishezon spaces" [35, page 331]. Of course we don't know how one could try to prove (or disprove) the existence of this infinite number of connected components. 


\section{References}

[1] D Allcock, Infinitely many hyperbolic Coxeter groups through dimension 19, Geom. Topol. 10 (2006) 737-758 MR2240904

[2] J Amorós, M Burger, K Corlette, D Kotschick, D Toledo, Fundamental groups of compact Kähler manifolds, Math. Surveys and Monogr. 44, Amer. Math. Soc. (1996) MR1379330

[3] M F Atiyah, N Hitchin, I M Singer, Self-duality in four-dimensional Riemannian geometry, Proc. Roy. Soc. London Ser. A 362 (1978) 425-461 MR506229

[4] A L Besse, Einstein manifolds, Ergebnisse der Math. und ihrer Grenzgebiete (3) 10, Springer, Berlin (1987) MR867684

[5] F A Bogomolov, On Guan's examples of simply connected non-Kähler compact complex manifolds, Amer. J. Math. 118 (1996) 1037-1046 MR1408498

[6] M Boileau, J Porti, Geometrization of 3-orbifolds of cyclic type, Astérisque 272, Soc. Math. France (2001) MR1844891 Appendix A by M Heusener and Porti

[7] T Bridgeland, A King, M Reid, The McKay correspondence as an equivalence of derived categories, J. Amer. Math. Soc. 14 (2001) 535-554 MR1824990

[8] C H Clemens, Double solids, Adv. in Math. 47 (1983) 107-230 MR690465

[9] J Davidov, G Grantcharov, O Muškarov, Curvature properties of the Chern connection of twistor spaces, Rocky Mountain J. Math. 39 (2009) 27-48 MR2476800

[10] J Davidov, O Muškarov, G Grantcharov, Almost complex structures on twistor spaces, from: "Almost complex structures (Sofia, 1992)", (K Sekigawa, S Dimiev, editors), World Sci. Publ., River Edge, NJ (1994) 113-149 MR1375930

[11] M W Davis, A hyperbolic 4-manifold, Proc. Amer. Math. Soc. 93 (1985) 325-328 MR770546

[12] J Eells, S Salamon, Twistorial construction of harmonic maps of surfaces into fourmanifolds, Ann. Scuola Norm. Sup. Pisa Cl. Sci. (4) 12 (1985) 589-640 (1986) MR848842

[13] J Fine, D Panov, The holomorphic geometry of flat bundles over hyperbolic 3manifolds, in preparation

[14] J Fine, D Panov, Simply connected symplectic 6-manifolds with vanishing first Chern class and arbitrarily large Betti numbers, in preparation

[15] J Fine, D Panov, Symplectic Calabi-Yau manifolds, minimal surfaces and the hyperbolic geometry of the conifold, J. Differential Geom. 82 (2009) 155-205 MR2504773

[16] R Friedman, Simultaneous resolution of threefold double points, Math. Ann. 274 (1986) 671-689 MR848512

[17] É Ghys, Déformations des structures complexes sur les espaces homogènes de SL(2, C), J. Reine Angew. Math. 468 (1995) 113-138 MR1361788 
[18] C M Gordon, Some aspects of classical knot theory, from: "Knot theory (Proc. Sem., Plans-sur-Bex, 1977)", (J-C Hausmann, editor), Lecture Notes in Math. 685, Springer, Berlin (1978) 1-60 MR521730

[19] P Griffiths, J Harris, Principles of algebraic geometry, Wiley Classics Library, Wiley, New York (1994) MR1288523 Reprint of the 1978 original

[20] M Gromov, Pseudoholomorphic curves in symplectic manifolds, Invent. Math. 82 (1985) 307-347 MR809718

[21] D Guan, Examples of compact holomorphic symplectic manifolds which are not Kählerian. II, Invent. Math. 121 (1995) 135-145 MR1345287

[22] S Helgason, Differential geometry, Lie groups, and symmetric spaces, Pure and Applied Math. 80, Academic Press, New York (1978) MR514561

[23] A T Huckleberry, J Winkelmann, Subvarieties of parallelizable manifolds, Math. Ann. 295 (1993) 469-483 MR1204832

[24] V A Iskovskikh, Y G Prokhorov, Fano varieties, from: "Algebraic geometry. V", Ency. Math. Sci. 47, Springer, Berlin (1999) 1-247 MR1668579

[25] C LeBrun, $\mathcal{H}$-space with a cosmological constant, Proc. Roy. Soc. London Ser. A 380 (1982) 171-185 MR652038

[26] C LeBrun, On complete quaternionic-Kähler manifolds, Duke Math. J. 63 (1991) 723-743 MR1121153

[27] T-J Li, Y Ruan, Symplectic birational geometry arXiv:0906.3265v1

[28] P Lu, G Tian, The complex structures on connected sums of $S^{3} \times S^{3}$, from: "Manifolds and geometry (Pisa, 1993)", (P de Bartolomeis, F Tricerri, E Vesentini, editors), Sympos. Math. XXXVI, Cambridge Univ. Press (1996) 284-293 MR1410077

[29] D McDuff, Examples of simply-connected symplectic non-Kählerian manifolds, J. Differential Geom. 20 (1984) 267-277 MR772133

[30] D McDuff, The structure of rational and ruled symplectic 4-manifolds, J. Amer. Math. Soc. 3 (1990) 679-712 MR1049697

[31] K Niederkrüger, F Pasquotto, Desingularization of orbifolds obtained from symplectic reduction at generic coadjoint orbits, Int. Math. Res. Not. (2009) 4463-4479 MR2558338

[32] K Niederkrüger, F Pasquotto, Resolution of symplectic cyclic orbifold singularities, J. Symplectic Geom. 7 (2009) 337-355 MR2534189

[33] L Potyagailo, E Vinberg, On right-angled reflection groups in hyperbolic spaces, Comment. Math. Helv. 80 (2005) 63-73 MR2130566

[34] J G Ratcliffe, S T Tschantz, On the Davis hyperbolic 4-manifold, Topology Appl. 111 (2001) 327-342 MR1814232 
[35] M Reid, The moduli space of 3-folds with $K=0$ may nevertheless be irreducible, Math. Ann. 278 (1987) 329-334 MR909231

[36] A G Reznikov, Symplectic twistor spaces, Ann. Global Anal. Geom. 11 (1993) 109-118 MR1225431

[37] S M Salamon, Minimal surfaces and symmetric spaces, from: "Differential geometry (Santiago de Compostela, 1984)", (L A Cordero, editor), Res. Notes in Math. 131, Pitman, Boston (1985) 103-114 MR864861

[38] S Smale, A Vietoris mapping theorem for homotopy, Proc. Amer. Math. Soc. 8 (1957) 604-610 MR0087106

[39] I Smith, R P Thomas, S-T Yau, Symplectic conifold transitions, J. Differential Geom. 62 (2002) 209-242 MR1988503

[40] C H Taubes, The Seiberg-Witten and Gromov invariants, Math. Res. Lett. 2 (1995) 221-238 MR1324704

[41] G Tian, Smoothing 3-folds with trivial canonical bundle and ordinary double points, from: "Essays on mirror manifolds", (S-T Yau, editor), Int. Press, Hong Kong (1992) 458-479 MR1191437

[42] C T C Wall, Classification problems in differential topology. V. On certain 6-manifolds, Invent. Math. 1 (1966), 355-374; corrigendum, ibid 2 (1966) 306 MR0215313

[43] N R Wallach, Symplectic geometry and Fourier analysis, Lie groups: History, frontiers and applications V, Math Sci Press, Brookline, MA (1977) MR0488148 With an appendix on quantum mechanics by R Hermann

[44] J-Y Welschinger, Open strings, Lagrangian conductors and Floer functor arXiv: $0812.0276 \mathrm{v} 1$

Départment de Mathématique, Université Libre de Bruxelles

CP218, Boulevard du Triomphe, Bruxelles 1050, Belgium

Department of Mathematics, Imperial College London

South Kensington Campus, London SW7 2AZ, United Kingdom

joel.fine@ulb.ac.be, d.panov@imperial.ac.uk

Proposed: Simon Donaldson

Received: 26 October 2009

Seconded: Ron Stern, Gang Tian

Revised: 16 March 2010 